\title{
FIGS-Faint Infrared Grism Survey: Description and Data Reduction
}

Norbert Pirzkal $^{1}$, Sangeeta Malhotra ${ }^{2,3}$ (D), Russell E. Ryan ${ }^{1}$, Barry Rothberg ${ }^{4,5}$ (1), Norman Grogin ${ }^{1}$ (i), Steven L. Finkelstein ${ }^{6}$ (1), Anton M. Koekemoer ${ }^{1}$ (D) James Rhoads ${ }^{2,3}$, Rebecca L. Larson ${ }^{6}$ (1) , Lise Christensen ${ }^{7}$ (1), Andrea Cimatti ${ }^{8,9}$ (1) , Ignacio Ferreras ${ }^{10}$, Jonathan P. Gardner ${ }^{3}$, Caryl Gronwall ${ }^{11,12}$, Nimish P. Hathi ${ }^{1,13}$, Pascale Hibon ${ }^{14}$ (1) , Bhavin Joshi ${ }^{2}$, Harald Kuntschner ${ }^{15}$, Gerhardt R. Meurer ${ }^{16}$, Robert W. O'Connell ${ }^{17}$, Goeran Oestlin ${ }^{18}$, Anna Pasquali ${ }^{19}$, John Pharo ${ }^{2}$, Amber N. Straughn ${ }^{10}$, Jeremy R. Walsh ${ }^{15}$, Darach Watson ${ }^{7}$ (D) Rogier A. Windhorst ${ }^{2}$ (D), Nadia L Zakamska ${ }^{20}$ (D), and Andrew Zirm ${ }^{21}$ (1)

${ }^{1}$ Space Telescope Science Institute, Baltimore, MD, 21210, USA

${ }^{2}$ Arizona State University, Tempe, AZ, 85287, USA

${ }^{3}$ NASAs Goddard Space Flight Center, Astrophysics Science Division, Code 660, Greenbelt, MD 20771 USA

${ }^{4}$ Large Binocular Telescope Observatory, University of Arizona, AZ, 85721, USA

${ }^{5}$ George Mason University, Fairfax, VA 22030, USA

${ }^{6}$ University of Texas at Austin, Austin, TX, 78712, USA

${ }^{7}$ Dark Cosmology Centre, Niels Bohr Institute, University of Copenhagen, DK-2100, Denmark

${ }^{8}$ University of Bologna, Department of Physics and Astronomy, Via Gobetti 93/2, I-40129, Bologna, Italy

INAF-Osservatorio Astrofisico di Arcetri, Largo E. Fermi 5, I-50125, Firenze, Italy

${ }_{10}^{10}$ Mullard Space Science Laboratory, University College London, Holmbury St. Mary, Dorking, Surrey RH5 6NT, UK

${ }^{11}$ Department of Astronomy and Astrophysics, The Pennsylvania State University, University Park, PA 16802, USA

${ }^{12}$ Institute for Gravitation and the Cosmos, The Pennsylvania State University, University Park, PA 16802, USA

${ }^{13}$ Aix Marseille Université, CNRS, LAM, Marseille, F-13388, France

${ }_{15}^{14}$ Gemini Observatory, Southern Operations, La Serena, Chile

${ }^{15}$ European Southern Observatory, Garching, D-85748, Germany

${ }^{16}$ International Centre for Radio Astronomy Research, The University of Western Australia, Crawley WA 6009, Australia

${ }^{17}$ The University of Virginia, Charlottesville, VA, 22904-4325, USA

${ }^{18}$ Stockholm University, Stockholm, SE-10691, Sweeden

${ }^{19}$ Astronomisches Rechen Institut, Zentrum für Astronomie der Universität Heidelberg, D-69120 Heidelberg, Germany

${ }^{20}$ Department of Physics and Astronomy, Johns Hopkins University, Baltimore MD 21218, USA

${ }^{21}$ University of Copenhagen, Niels Bohr Institute, Kbenhavn, DK-2100, Denmark

Received 2017 April 20; revised 2017 June 16; accepted 2017 June 16; published 2017 September 1

\begin{abstract}
The Faint Infrared Grism Survey (FIGS) is a deep Hubble Space Telescope (HST) WFC3/IR (Wide Field Camera 3 Infrared) slitless spectroscopic survey of four deep fields. Two fields are located in the Great Observatories Origins Deep Survey-North (GOODS-N) area and two fields are located in the Great Observatories Origins Deep Survey-South (GOODS-S) area. One of the southern fields selected is the Hubble Ultra Deep Field. Each of these four fields were observed using the WFC3/G102 grism $(0.8 \mu \mathrm{m}-1.15 \mu \mathrm{m}$ continuous coverage $)$ with a total exposure time of 40 orbits $(\approx 100$ kilo-seconds) per field. This reaches a $3 \sigma$ continuum depth of $\approx 26 \mathrm{AB}$ magnitudes and probes emission lines to $\sim 10^{-17} \mathrm{erg} \mathrm{s}^{-1} \mathrm{~cm}^{-2}$. This paper details the four FIGS fields and the overall observational strategy of the project. A detailed description of the Simulation Based Extraction (SBE) method used to extract and combine over 10,000 spectra of over 2000 distinct sources brighter than $m_{F 105 \mathrm{~W}}=26.5$ mag is provided. High fidelity simulations of the observations is shown to significantly improve the background subtraction process, the spectral contamination estimates, and the final flux calibration. This allows for the combination of multiple spectra to produce a final high quality, deep, 1D spectra for each object in the survey.
\end{abstract}

Key words: galaxies: general - surveys - techniques: spectroscopic

\section{Introduction}

The study of distant galaxies is dependent on how well one can reliably derive accurate redshifts. The most accurate method relies on spectroscopic emission or absorption lines, followed by broad spectroscopic features such as the $4000 \AA$ and Lyman breaks. This is particularly important when deploying large surveys to catalog and discern the properties of objects as a function of cosmic epoch. Absent spectroscopy, photometric methods (photo- $z$ ) using color selection, often supplemented by theoretical methods such as fitting spectral energy distribution (SED) templates to the data, can provide rough redshift estimates. The power and relative accuracy of photometric methods are dependent on the sample size used, robust statistical analysis, the quality (and appropriateness) of the input model SEDs or empirical template spectra used, and properly calibrating techniques using spectroscopic data sets. The smaller the sample size, the less reliable photo- $z$ methods are, and thus one should proceed with extreme caution when used for individual objects (as noted in Sawicki et al. 1997; Liu \& Green 1998; Pirzkal et al. 2013b). Therefore, spectroscopic follow-up is always required to cull samples of false positives and, for example, should always be the final arbiter in rejecting or supporting claims for the most distant objects detected.

However, spectroscopic observations are not without their own set of complications. Extracting object parameters other than redshift alone is extremely expensive in terms of aperture and time required, particularly in probing the earliest epochs of galaxy formation. This is of course because more signal-tonoise $(\mathrm{S} / \mathrm{N})$ is required to detect continuum, and an even higher $\mathrm{S} / \mathrm{N}$ is required to detect absorption lines or separate close emission lines, particularly at wavelengths greater than $0.8 \mu \mathrm{m}$, where telluric sky emission and atmospheric absorption greatly affect observations. 

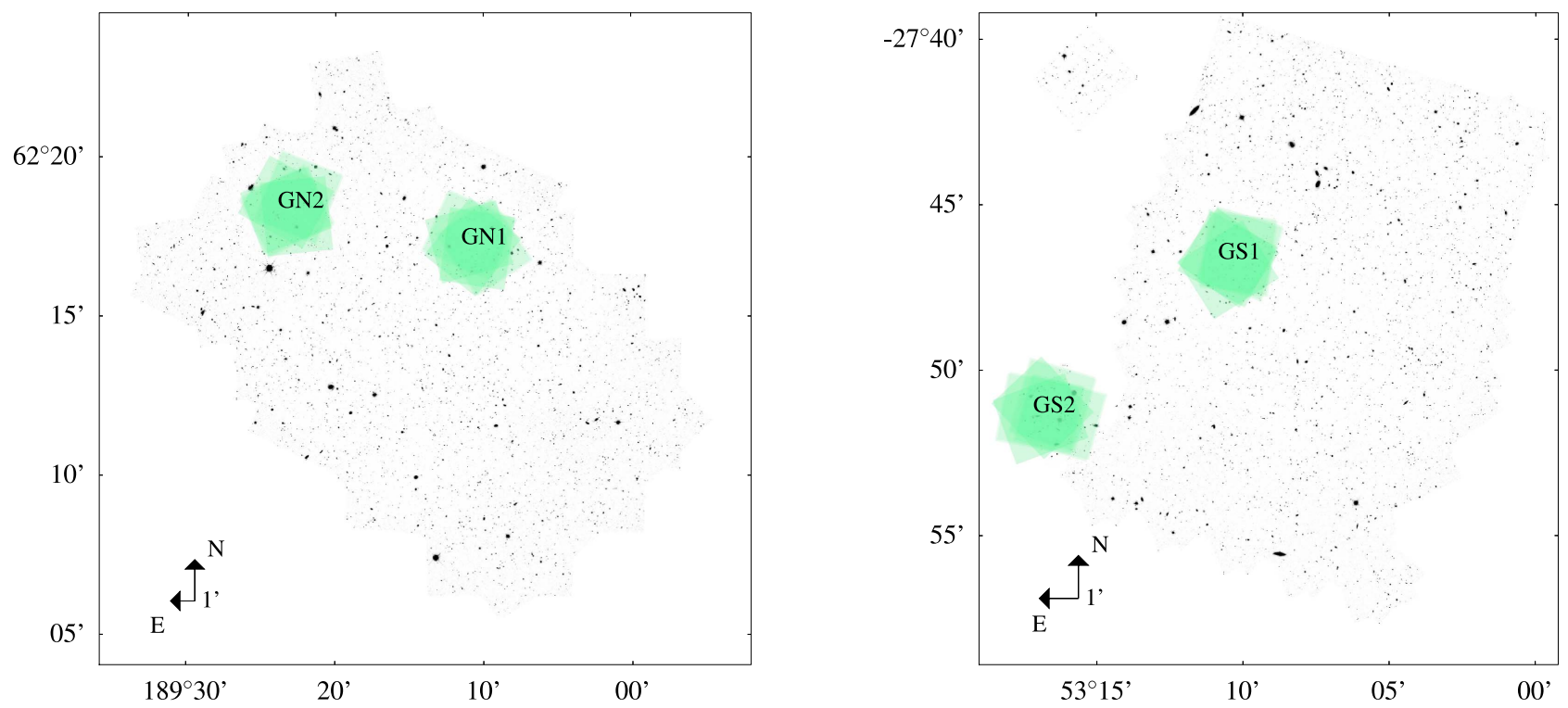

Figure 1. Left panel: location of the FIGS N Fields (green) with respect to the GOODS-N field. Right panel: location of the FIGS S Fields (green) with respect to the GOODS-S field. The GS1 field is at the same position as the HUDF field. The GS2 field is located at the UDF-PAR2 field. The GOODS-N and GOODS-S mosaics are also shown. F125W mosaics are shown.

Table 1

Coordinates and Exposure Time of the Four FIGS Fields

\begin{tabular}{|c|c|c|c|c|c|}
\hline $\begin{array}{l}\text { Field } \\
\text { Name }\end{array}$ & R.A. & Decl. & $\begin{array}{c}\text { Position } \\
\text { Angles (PA) }\end{array}$ & $\begin{array}{c}\text { Number } \\
\text { of Exposures }\end{array}$ & $\begin{array}{c}\text { Total } \\
\text { Exposure Time }\end{array}$ \\
\hline$\overline{\mathrm{GN} 1}$ & $12^{\mathrm{h}} 36^{\mathrm{m}} 42^{\mathrm{s}} \cdot 56$ & $62^{\mathrm{d}} 17^{\mathrm{m}} 16^{\mathrm{s}} .89$ & $-164,-128,-98,-56,156$ & 320 & 101120 \\
\hline GN2 & $12^{\mathrm{h}_{3}} 37^{\mathrm{m}_{3}} 32^{\mathrm{s}} .04$ & $62^{\mathrm{d}} 18^{\mathrm{m}} 26^{\mathrm{s}} .06$ & $-158,-152,-83,68,151$ & 288 & 103823 \\
\hline GS2 & $03^{\mathrm{h}} 33^{\mathrm{m}} 06^{\mathrm{s}} .76$ & $-27^{\mathrm{d}} 51^{\mathrm{m}} 16^{\mathrm{s}} \cdot 56$ & $-159,-15,73,133,169$ & 288 & 98822 \\
\hline
\end{tabular}

Technical issues, such as proper slit alignment, slit losses, fitting multiple slits on a multi-object mask or multiple fiber placement, size and efficiency of integral field unit spectrographs, flux and wavelength calibration, atmospheric extinction at short wavelengths, and the ever increasing atmospheric absorption and thermal sky emission at longer wavelengths, play a significant role in affecting the viability of spectroscopic surveys. The more dispersed the light is, the more expensive the survey (i.e., the longer the integration times and the larger the collecting surface needed). A work-around for this is not new or novel. Low-resolution wide-field slitless spectroscopy for detecting faint targets was first developed over 120 years ago at the Lick Observatory with the Slitless Quartz Spectrograph for use on the 36" Crossley Reflector (Palmer 1903). This was later refined for surveying the radial velocities of "extra-galactic nebulae" (Mayall 1936). These surveys focused primarily on UV/Optical $(\lambda<0.5 \mu \mathrm{m})$ low-resolution spectroscopy of emission lines. Many surveys continued over the decades, e.g., Markarian (1967), Smith (1975), MacAlpine et al. (1977), and Wasilewski (1983), helping to discover and catalog quasars and emission-line galaxies, as well as the search for young stars and their places of formation, within our own Galaxy, e.g., Dahm (2005). Grism surveys can be more efficient, requiring less observing time to reach a given $\mathrm{S} / \mathrm{N}$, than their slit/grating counterparts, and can be significantly more robust and reliable than photometric redshift surveys, e.g., Smith (1978) and Schmidt et al. (1986). Specifically, slitless spectroscopy does not suffer light loss compared to slit spectroscopy, grism surveys are significantly more efficient in collecting area, and, even with multi-object slit masks or fibers, there is a limit to the number of slits or fibers that can be placed on the sky within a field of view. Slitless grism (whether groundor space-based) observations are not hampered by these limitations. Furthermore, the low-resolution of grism surveys results in a gain in $\mathrm{S} / \mathrm{N}$. This is relevant for all telescope aperture sizes. However, as efficient as these surveys are, the push to higher redshifts, fainter targets, and multiple emission lines to extract physical parameters beyond redshift alone, cannot compete with telluric limitations. At longer wavelengths the sky-brightness and sky emission lines, along with the limitations of what the Earth's atmosphere absorbs severely limits slitless spectroscopic surveys. With the launch of HST and the installation of improved instrumentation, grism survey work has seen a renaissance in the last two decades. This resurgence began with the Near Infrared Camera and Multi-Object Spectrometer (NICMOS), which included surveys such as McCarthy et al. (1999), followed by the Advanced Camera for Surveys (ACS), which, in addition to pointed surveys, made possible grism surveys parallel to $H S T$ primary observations (APPLES: Pasquali et al. 2003, GRAPES: Pirzkal et al. 2004, and PEARS: Pirzkal et al. 2009), 3D-HST: Momcheva et al. (2016) and GLASS: Treu et al. (2015). With the addition of the Wide Field Camera 3 and its ability to cover $0.2-1.6 \mu \mathrm{m}$ (split over two grisms), wide-field grism observations are cornerstone 

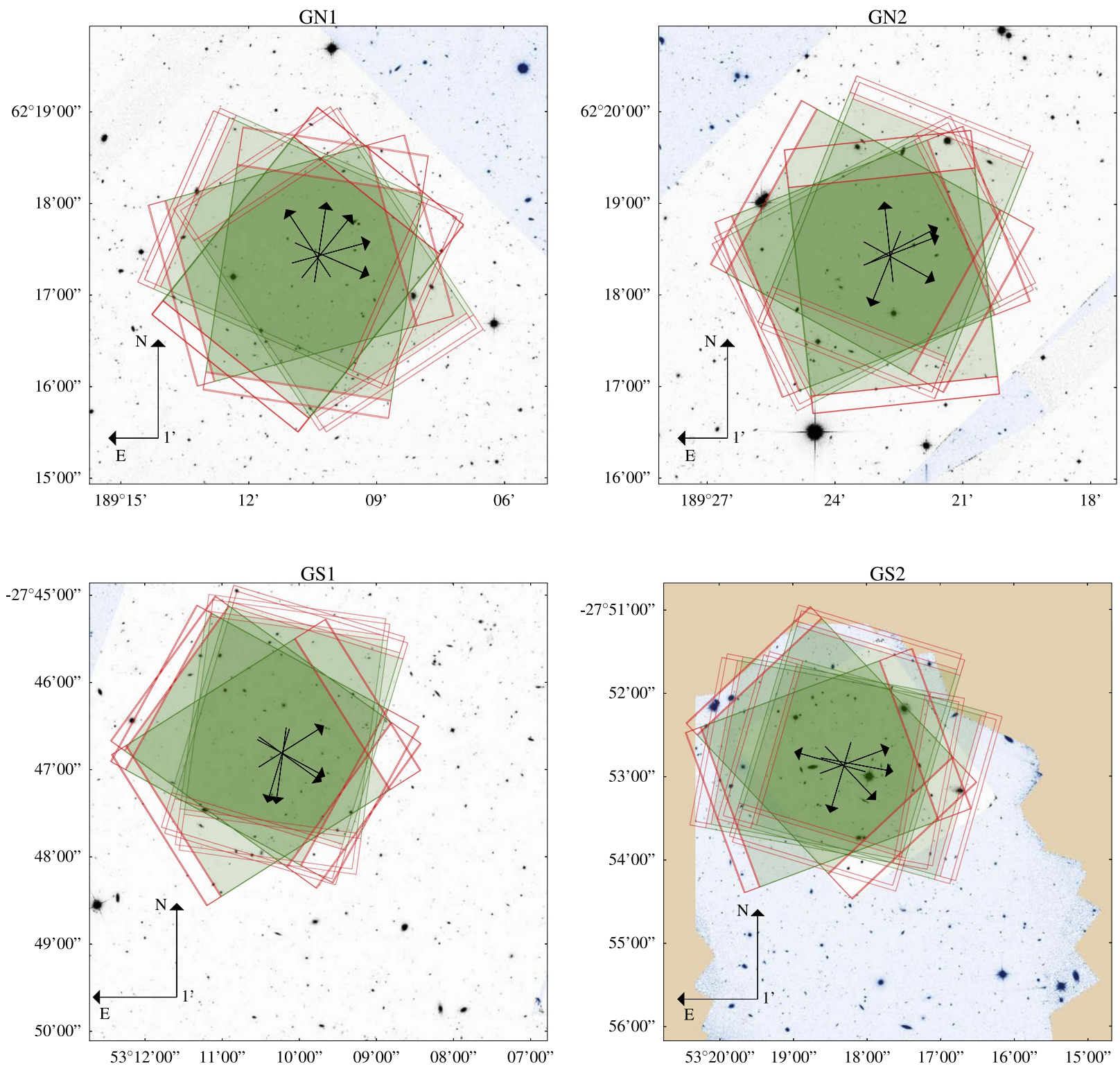

Figure 2. Four FIGS fields. Each of the four fields were observed at five different position angles, as shown by the black arrows at the center of each field. The regions covered by the grism are shown in green, while regions near the field of view, in which objects outside of the field of view could still result in dispersed orders, are delineated using red lines. The gray mosaic shows areas with existing F105W imaging. Areas with only ACS $z$-band data and no F105W data are shown in blue. Parts of the fields where no imaging was available to determine the existence of contaminating objects are shown in orange. In these plots, north is up and the footprints of individual G102 observations are shown in green and can be directly compared to Figure 1.

data products for large surveys such as the WFC3 Infrared Spectroscopic Parallel Survey (WISPS: Atek et al. 2010), 3DHST (Brammer et al. 2012), the Grism-Lens Amplified Survey from Space (GLASS: Schmidt et al. 2014), and the Faint Infrared Grism Survey (FIGS: described herein). Moving deep grism surveys from the ground to space has led to a vast improvement in our ability to detect fainter targets, opening a new parameter space for both the most distant objects and lower mass ranges.

In this paper, we present the data reduction and spectral extraction for the cycle 22 Treasury program: FIGS (Proposal ID: 13779, PI: S. Malhotra). FIGS was awarded 160 orbits with the WFC3/IR instrument to survey four distinct fields with five Position Angles (PAs) for each field using the G102 grism to a depth of $m_{F 105 W} \approx 26$. FIGS is the deepest HST grism survey to date. FIGS data are ideal for constraining cosmic reionization at $z \gtrsim 7$ through detection of Ly $\alpha$ emission (e.g., Malhotra \&
Rhoads 2004; Stern et al. 2005; Tilvi et al. 2016); probing the star-formation histories for red sequence/blue cloud/green valley galaxies at $z \sim 2$ (e.g., Pasquali et al. 2006; Ferreras et al. 2009, 2012; Bedregal et al. 2013; Whitaker et al. 2013); examining the diversity among emission-line galaxies at $z \lesssim 2$ (e.g., Straughn et al. 2008; Pirzkal et al. 2013a; Atek et al. 2014); and providing an unbiased redshift census (e.g., Ryan et al. 2007; Brammer et al. 2012).

This paper is organized as follows. In Section 2, the survey motivation and design is explained. In Section 3, details regarding the data reduction, including object catalogs and spectral extraction are given. In Section 4, the combination of spectra at multiple position angles, $\mathrm{S} / \mathrm{N}$ calculations are explained, and representative examples for several types of astrophysical sources are provided. Throughout this work, magnitudes are provided in AB units (Oke \& Gunn 1983). 


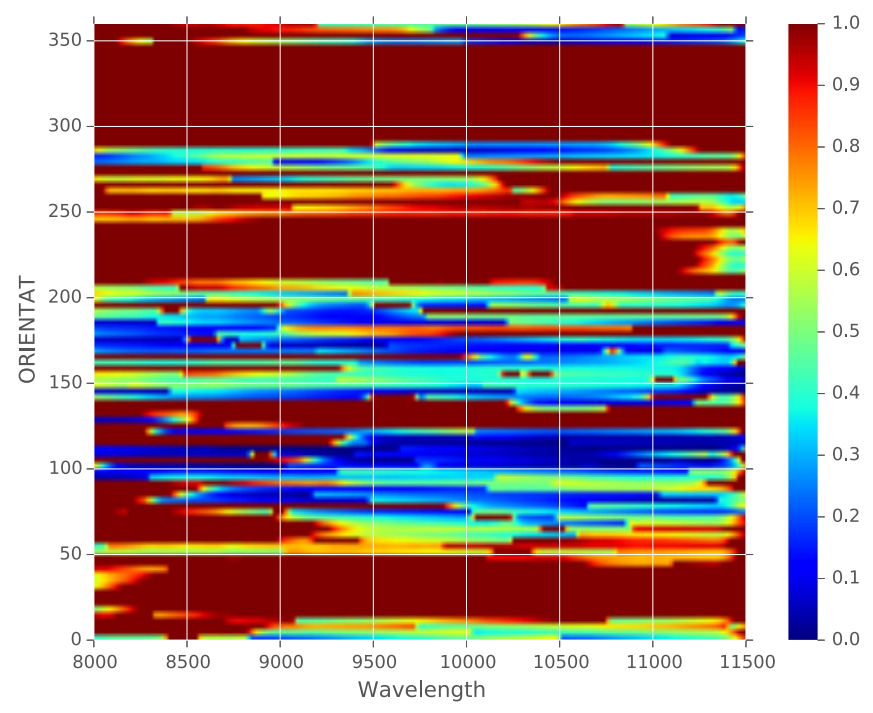

Figure 3. Example of one of the diagnostic plots used to determine the position angles (ORIENTAT) where a source of interest was the least contaminated. Regions of high contamination are shown in red while low contamination regions appear in blue. In this example, the lowest amount of contamination occurs at a position angle of $99^{\circ}$. This plot shows the fraction of the observed flux that is due to contamination as a function of position angle and wavelength. Plots such as this one were generated for each of our high-redshift $(z>6)$ candidates. We then manually selected specific position angles for each field by manually maximizing the number of some of the brighter high-z targets likely to be uncontaminated.

Table 2

FIGS SeXtractor Parameters Used to Generate the Cold and Hot Detection Catalogs

\begin{tabular}{lccc}
\hline \hline \multirow{2}{*}{$\begin{array}{l}\text { SeXtractor } \\
\text { Parameter }\end{array}$} & Cold & Hot & Super-hot \\
\cline { 2 - 4 } & 1.5 & 0.7 & 0.35 \\
\hline DETECT_THRESH & 28 & 28 & 14 \\
DETECT_MINAREA & 8 & 32 & 32 \\
DEBLEND_NTHRESH & 0.01 & 0.0001 & 0.0001 \\
DEBLEND_MINCONT & top-hat & Gaussian & Gaussian \\
Filter $(9 \times 9$ pixel $)$ & & & \\
\hline
\end{tabular}

\section{Survey Design}

FIGS is designed to maximize coverage and depth of field, while striving to reduce contamination and spurious detections by leveraging multiple PAs. The fields selected were based upon already available photometric data. Four pointings were selected for the FIGS observations, two in the GOODS-North region and two in the GOODS-South region (Table 1 and Figures 1 and 2). All four were chosen to maximize the number of high-redshift $(z>6)$ candidates within the WFC3/IR grism field of view $(69,21,144$, and 38 candidates in GN1, GN2, GS1, and GS2, respectively). These candidates were selected via SED fitting to the available $H S T$ deep multi-filter broadband imaging from $B$ band through to $H$ band (Finkelstein et al. 2015). These broadband exposures came from several different programs, including GOODS (Giavalisco et al. 2004), CANDELS (Grogin et al. 2011; Koekemoer et al. 2011), and successive HUDF campaigns (Beckwith et al. 2006). In the GOODS-South region, one pointing was situated within the HUDF, and the other was situated within an HUDF parallel field, slightly displaced from the GOODS area. The two GOODS-North fields were both within the CANDELS deep near-infrared area.
For each pointing location, the FIGS team simulated WFC3/ IR grism exposures as a function of HST roll angle, using the existing near-infrared images. We generated simulations with orientations ranging between $0^{\circ}$ and $360^{\circ}$ in steps of 0.5 . At each orientation, the ratio of contaminating flux to source flux was computed where the first-order spectrum of a particular high-redshift candidate was located. Plots such as the one shown in Figure 3 were generated to determine position angles where contamination was as low as possible (shown in blue). The simulations were then checked for dispersed source overlap, to identify five roll angles in each field that minimized grism contamination of the high-redshift candidates. The selected roll angles for each field are listed in Table 1. Some of these roll angles are close together but we ensured that at least three significantly different roll angles were obtained for each field. Four two-orbit visits were obtained for each roll angle with the G102 grism, resulting in 40 orbits per FIGS field and approximately $100 \mathrm{ksec}$ of exposure time per field.

The sequencing of grism and direct imaging exposures within each two-orbit $H S T$ visit was carefully tailored to protect the G102 exposures from illumination by the sunlit Earth limb. WFC3/IR observations at low limb-angle to the sunlit Earth are known to suffer from much higher and rapidly variable background, and are particularly sensitive to the air-glow of helium $\lambda 10830 \AA$ (Brammer et al. 2015). Once the observing window of an FIGS visit was fixed, we determined whether a given $H S T$ visit would be rising or setting over the sunlit Earth limb. This information was provided to us by our Program Coordinator (PC) at the Space Telescope Science Institute. We then placed the broadband F105W alignment exposure either before or after the G102 exposures, as appropriate, to take the brunt of the high-background portion of the orbit.

Because FIGS target fields are each a single WFC3/IR field of view, we opted for a minimally distributed dither pattern among observations within a single epoch. For each two-orbit visit, we initially chose the IR-DITHER-BLOB pattern (see Section C.2 of the WFC3 Instrument Handbook) of $\approx 3^{\prime \prime}$ between orbits, with small intra-orbit dithering $(\approx 0.3)$ for improved subpixel-phase sampling. The IR-DITHER-BLOB pattern has the added benefit of displacing the ACS/WFC parallel exposures by slightly more than the gap between the two ACS/WFC CCDs. Partway through the program's execution, we slightly expanded the inter-orbit dither pattern to match that adopted by the $3 \mathrm{D}-H S T$ program (Brammer et al. 2012), thereby better mitigating WFC3/IR self-persistence.

The portion of the WFC3/IR field of view for which the full G102 trace is available for all targets (as unvignetted by the detector edge) is not centered on the detector. To maximize the number of targets with full grism traces available in all five epochs, taken at varying $H S T$ roll angles, we further introduced an epoch-dependent dither offset $\left(\approx 17^{\prime \prime}\right)$ tailored to co-locate the full-trace region on the sky, regardless of HST orientation.

To maximize $\mathrm{S} / \mathrm{N}$ in the stack of dithered WFC3/IR grism exposures, the FIGS team initially chose a $100 \mathrm{~s}$ IR sampling sequence ("SPARS100") with 12-15 samples per exposure (exposure times between 1100 and 1400 s). Partway through the execution of the program, the team concluded that detector self-persistence was sufficiently severe (i.e., spectra of bright stars left a visible imprint in subsequent exposures at levels greater than $0.005 e^{-} \mathrm{s}^{-1}$ ) to merit a change to a $50 \mathrm{~s}$ sampling pattern ("SPARS50") with 12-15 samples per exposure (exposure times between 450 and $700 \mathrm{~s}$ ). Although this 

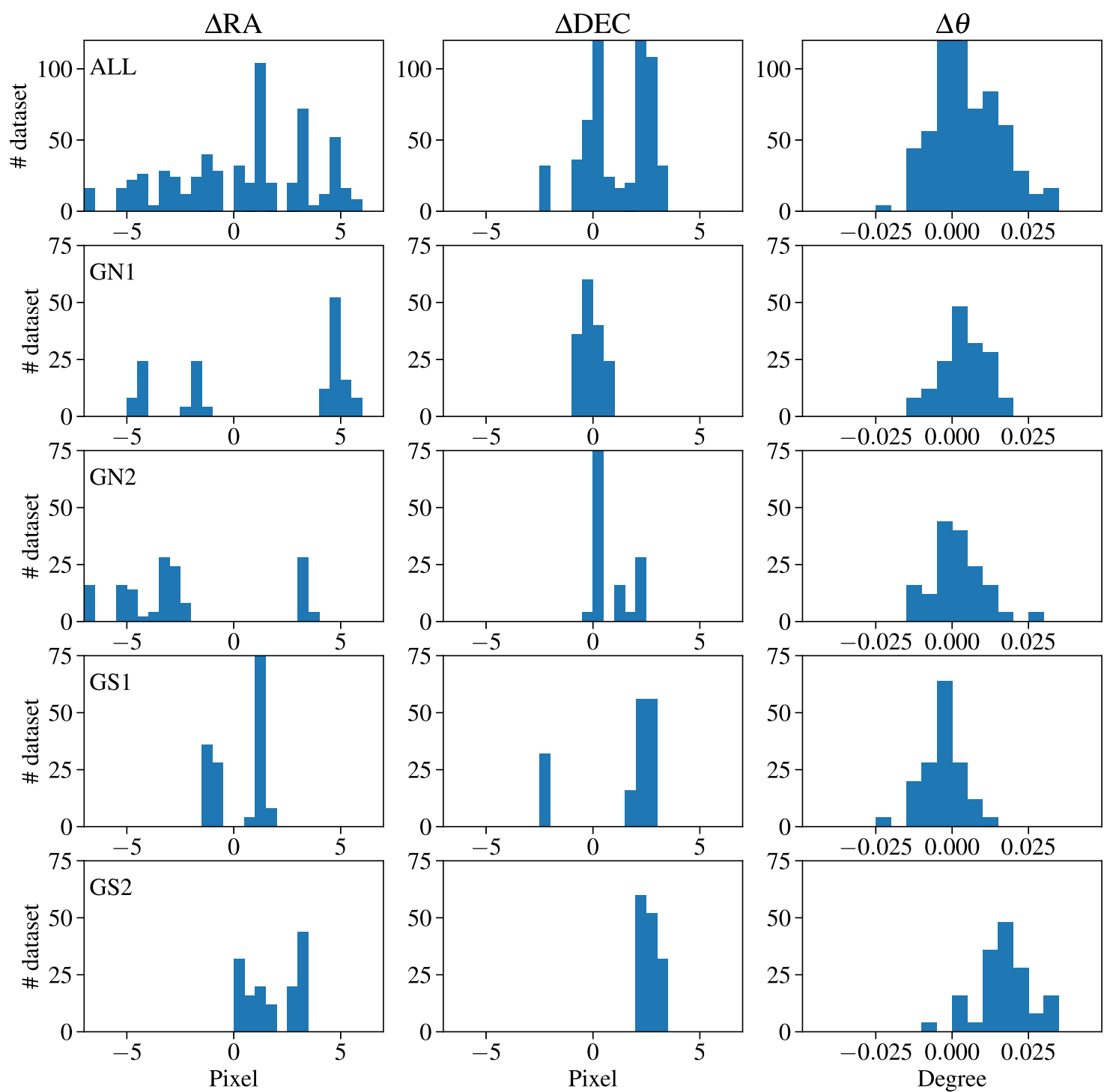

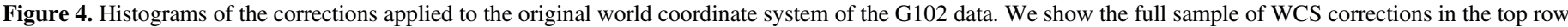

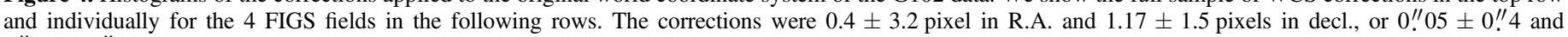
0 ! $15 \pm 0$." 19 , respectively. Rotations were within $\approx 0$. 01 .

doubled the number of exposures and resulted in a modest loss of cumulative IR exposure time (i.e., increased overhead), the overall sensitivity was improved by the reduction of selfpersistence within the image sequence, avoiding fake/ghost objects and spectra on subsequent, dithered exposures. The much shorter exposures for broadband (F105W) alignment used a $25 \mathrm{~s}$ sampling sequence ("SPARS25") with 11-13 samples per exposure.

\section{Data Reduction}

\subsection{Overview}

The FIGS G102 data were reduced in a manner that loosely follows the method used for the GRAPES and PEARS data (Pirzkal et al. 2004). However, the planning and execution of the observations were first based on accurate simulations, which then served as the the basis for the actual extraction of the data. Simulation Based Extraction (SBE) is a critical step to significantly improving source extraction. It is essential to first simulate the data so that the astrometric solution and the input object catalogs are validated. Furthermore, access to high fidelity simulations (Section 3.2.4) allows for fine-tuned background subtraction corrections (Section 3.2.6), detailed contamination estimates, as well as the appropriate application of object-specific sensitivity functions to each extracted spectrum (Section 4.2).

\subsection{Pre-processing}

\subsubsection{Field Mosaics}

For this project, we used the full-depth HST ACS and WFC3 mosaics of these fields, predominantly from CANDELS (Grogin et al. 2011; Koekemoer et al. 2011) and GOODS (Giavalisco et al. 2004), supplemented as needed by imaging from other HST programs (including the HUDF; Beckwith et al. 2006; Oesch et al. 2010; Ellis et al. 2013; Illingworth et al. 2013; Koekemoer et al. 2013). The filters used for detection and target selection in this project were primarily the F850LP in ACS (roughly equivalent to SDSS z), together with the F125W and F160W in WFC3 (roughly equivalent to $J$ and 

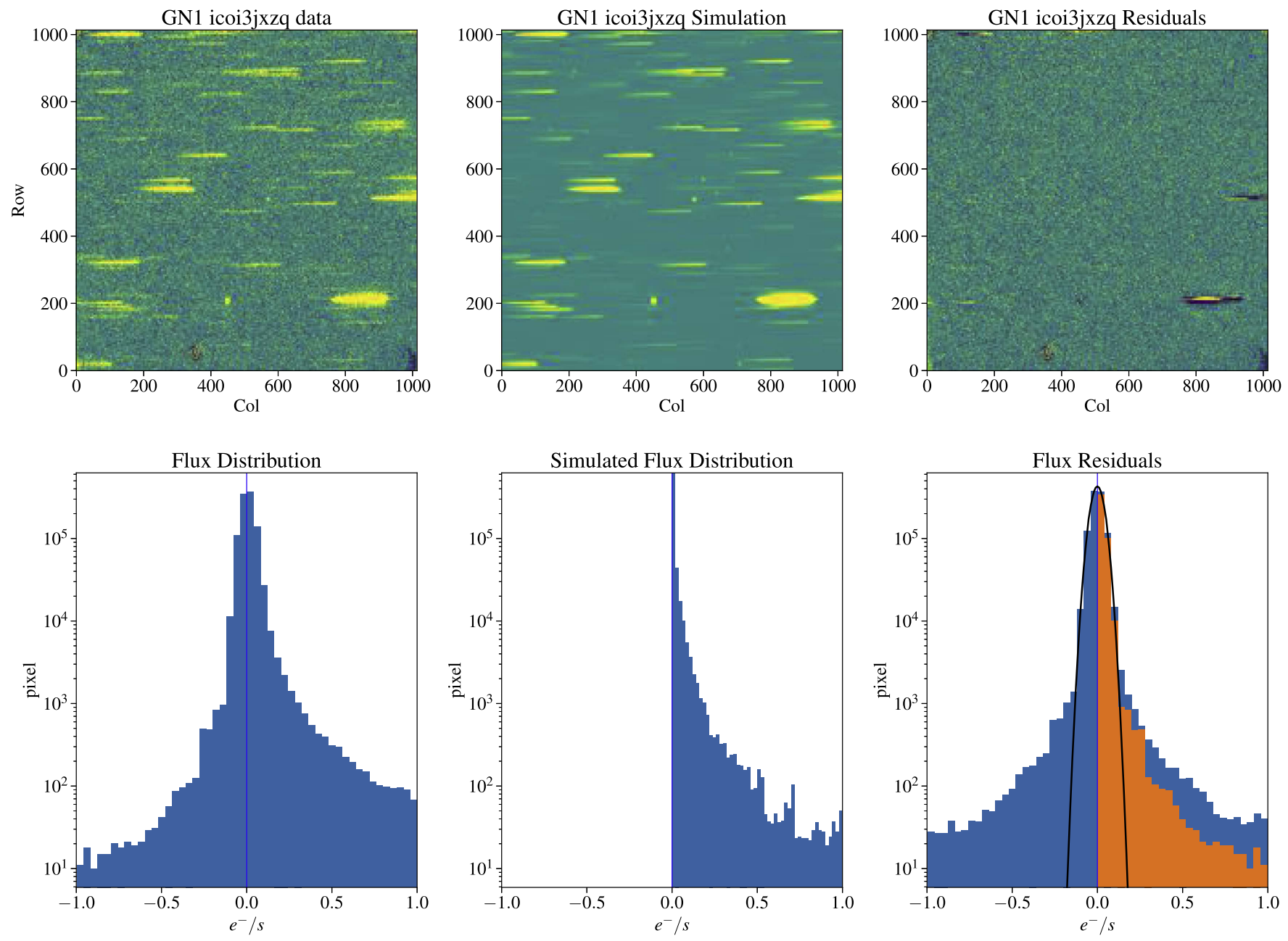

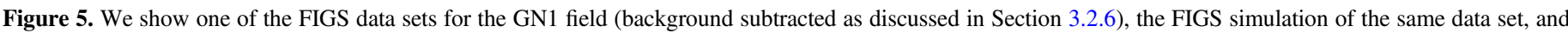

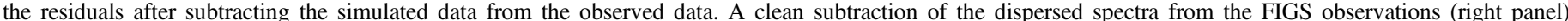

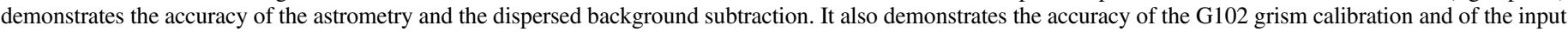

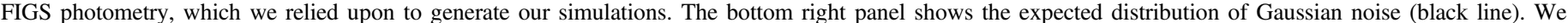

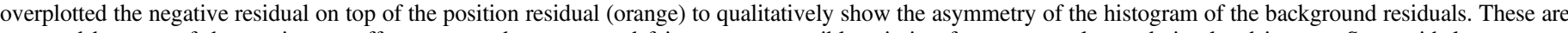

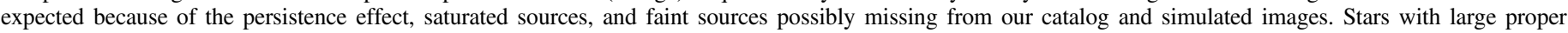
motions produce regions with larger positive and negative residuals in the top right panel.

$H$ bands, respectively). These mosaics have pixel scales of 30 mas/pixel for the F850LP and 60 mas/pixel for the F125W and F160W, and were used as the absolute astrometric reference for the new $H S T$ grism imaging that were obtained for these fields. They also served as detection images for all the spectroscopically detected sources. Further details are provided in Koekemoer et al. (2011) on the image combination and processing approaches that were used to produce these mosaics.

\subsubsection{Object Catalogs}

We created photometric catalogs using a custom version of Source Extractor (Bertin \& Arnouts 1996). Our modified version adds a buffer between the source and the local background cell and removes spurious sources associated with the distant wings of bright objects. Catalogs were generated independently in each of our four sub-fields, using a $10,000 \times 10,000$ pixel mosaic ( 30 mas per pixel and a size of 5 by 5 arcmin) centered on our grism pointings (see Section 3.2.1 for details on image reduction). We used Source Extractor in two-image mode, where the same detection image
(F125W) was used to measure photometry from all available $H S T$ filters. The choice of the $\mathrm{F} 125 \mathrm{~W}$ was predicated based on the fact that the F125W coverage of the FIGS fields is more complete and uniform than the F105W coverage. The choice of the $\mathrm{F} 125 \mathrm{~W}$ is also more appropriate to detect faint $z>8$ galaxies since these objects should drop out of F105W images. While, in theory, we might expect that some very low continuum galaxies with bright emission lines might be missing from our object extraction catalog, supplemental methods can be used to search for such sources (Straughn et al. 2008; Pirzkal et al. 2013a; N. Pirzkal et al. 2017, in preparation).

The filters used to provide supplemental photometric information to the $\mathrm{F} 125 \mathrm{~W}$ detection catalog were $\mathrm{F} 435 \mathrm{~W}$, F606W, F775W, and F850LP from the GOODS ACS survey, and F105W and F160W from the CANDELS WFC3/IR survey. Deep ACS/F814W imaging taken parallel to CANDELS was also used, as well as the shallow WFC3/IR F140W pre-imaging from 3DHST. In the GS1 field, which overlaps with the HUDF, we also made use of imaging from the HUDF, HUDF09 (Oesch et al. 2010), and UDF12 (Ellis et al. 2013). 

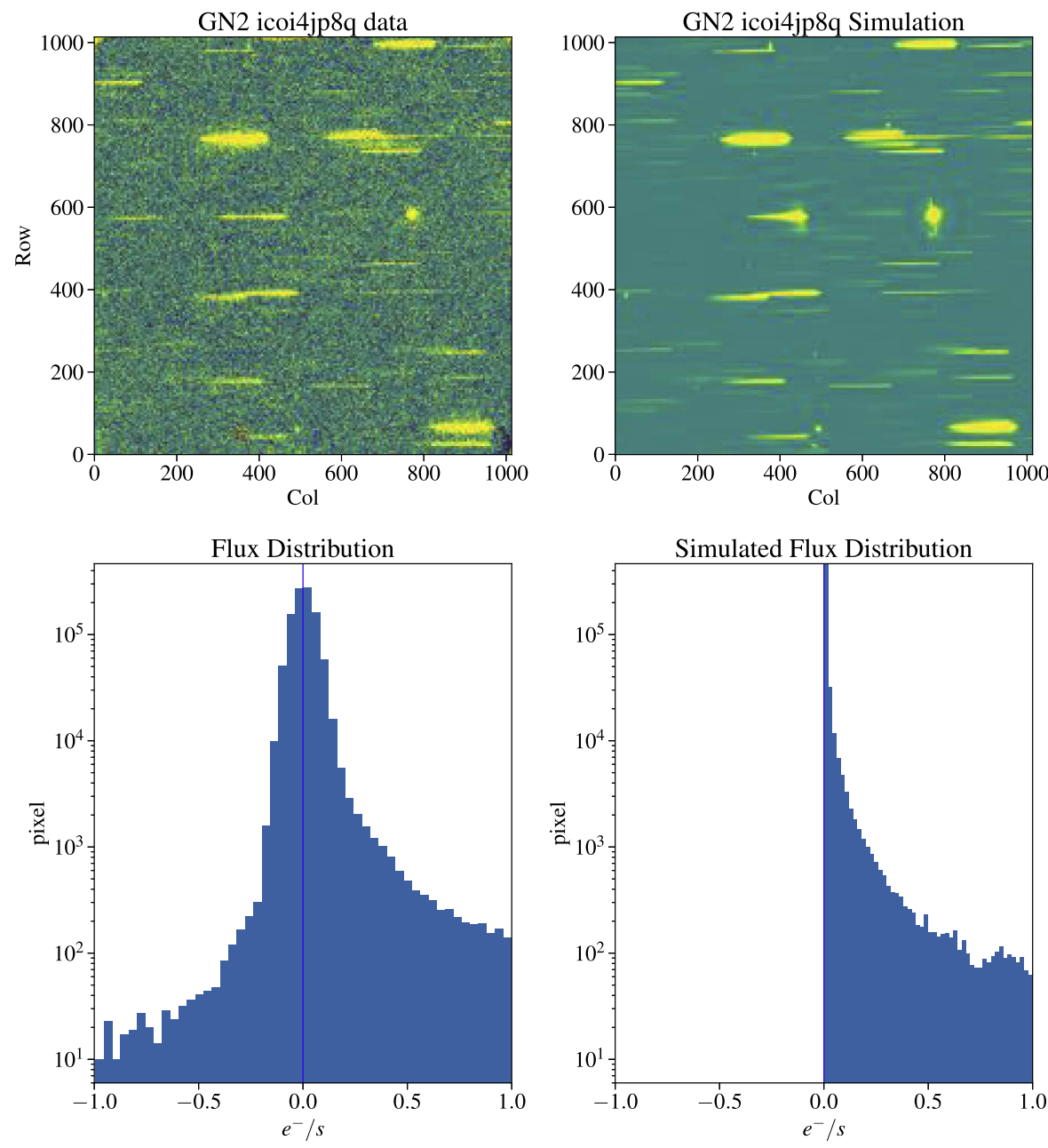

Figure 6. Same as Figure 5 but for the GN2 field.
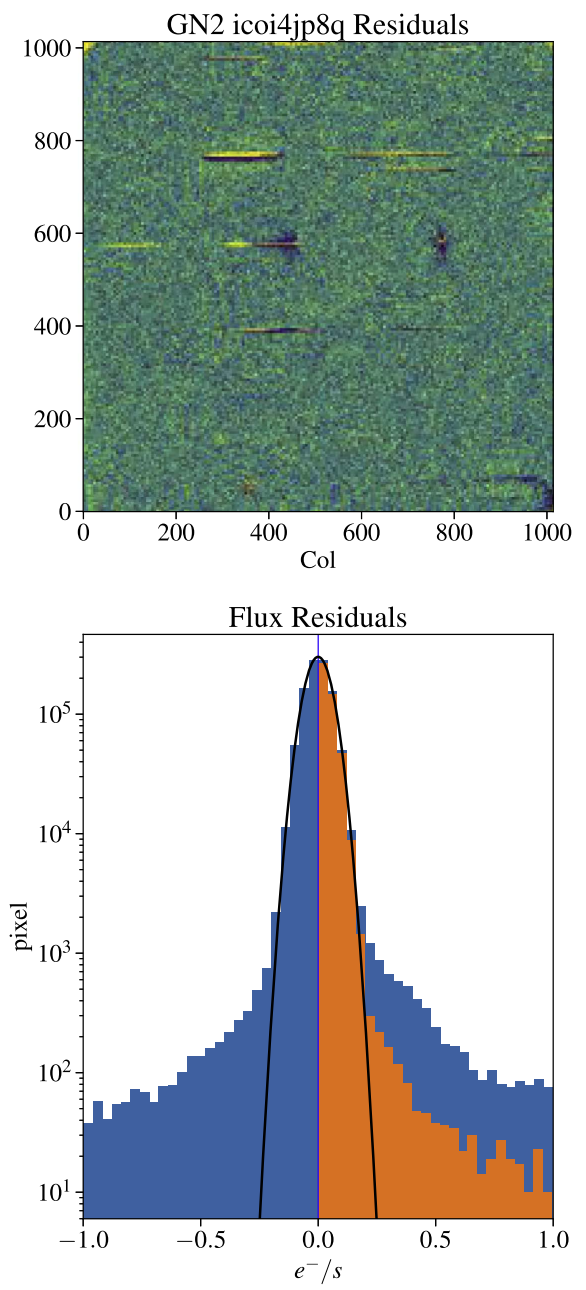

Because this catalog specifies the size and position of sources for extraction from the spectroscopic grism frames, the fidelity of the sources are important. For this reason, we elected to compose a combined photometric catalog, using both a "cold" catalog with conservative detection parameters, which keep large objects together, and a "hot" catalog with more aggressive detection parameters to ensure that we include faint sources. Table 2 lists the main extraction parameters used for both the cold and hot catalogs.

A minimum footprint size of 28 pixels (approximately equal to the number of pixels in the point-spread function at the redder WFC3/IR wavelengths) was used for Source Extractor. The detection and deblending parameters were tuned by inspecting both catalogs and ensuring that large galaxies remained a single object in the cold catalog, and very faint, yet likely real, objects were still detected in the hot catalog. Similar to the catalogs from Finkelstein et al. $(2010,2012,2015)$, we measure colors in small, elliptical Kron (Kron 1980) apertures with PHOT_AUTOPARAMS set to 1.2 and 1.7, and aperture corrections were derived in the F160W-band using the default MAG_AUTO parameters of 2.5 and 3.5 (which has been found to reliably return the total flux within $\sim 5 \%$ ). The default values of 2.5 and 3.5 correspond to an aperture that measures the total magnitude to within $6 \%$ accuracy. However, this aperture does not calculate colors with the optimal $\mathrm{S} / \mathrm{N}$, as it includes many sky pixels (a necessary trade-off to accurately measure the total flux). Finkelstein et al. (2012) showed that using smaller ellipses with PHOT_AUTOPARAMS set to 1.2 and 1.7 more accurately recovered colors for simulated sources (see also Bouwens et al. 2007). We therefore measure colors in these smaller apertures, but derive an aperture correction to the F160W-band as the ratio of the flux in the larger-to-smaller Kron apertures.

These aperture corrections can fail for objects near to very bright sources, as their Kron radii may not be reliably derived. In rare cases where aperture correction had non-physical negative values, visual inspection showed that these objects were close to brighter objects. For these circumstance, we applied statistical aperture corrections taken as the median aperture corrections for sources of similar fluxes $( \pm 10 \%)$.

This process resulted in a complete hot and cold catalog, containing fluxes for all objects in each catalog in each filter. These catalogs were then merged into a final catalog. However, objects in the hot catalog were only included if their central pixel had a value of zero in the cold catalog segmentation map (i.e., it did not lie within the isophotal region of a cold catalog object). For this comparison, the cold catalog segmentation map was dilated to slightly increase the area of each object (this was only applied during the merging process and did not affect the photometry). The combined catalogs were then visually inspected to identify and remove potential non-real objects, such as diffraction spikes from bright objects, or noise spikes 

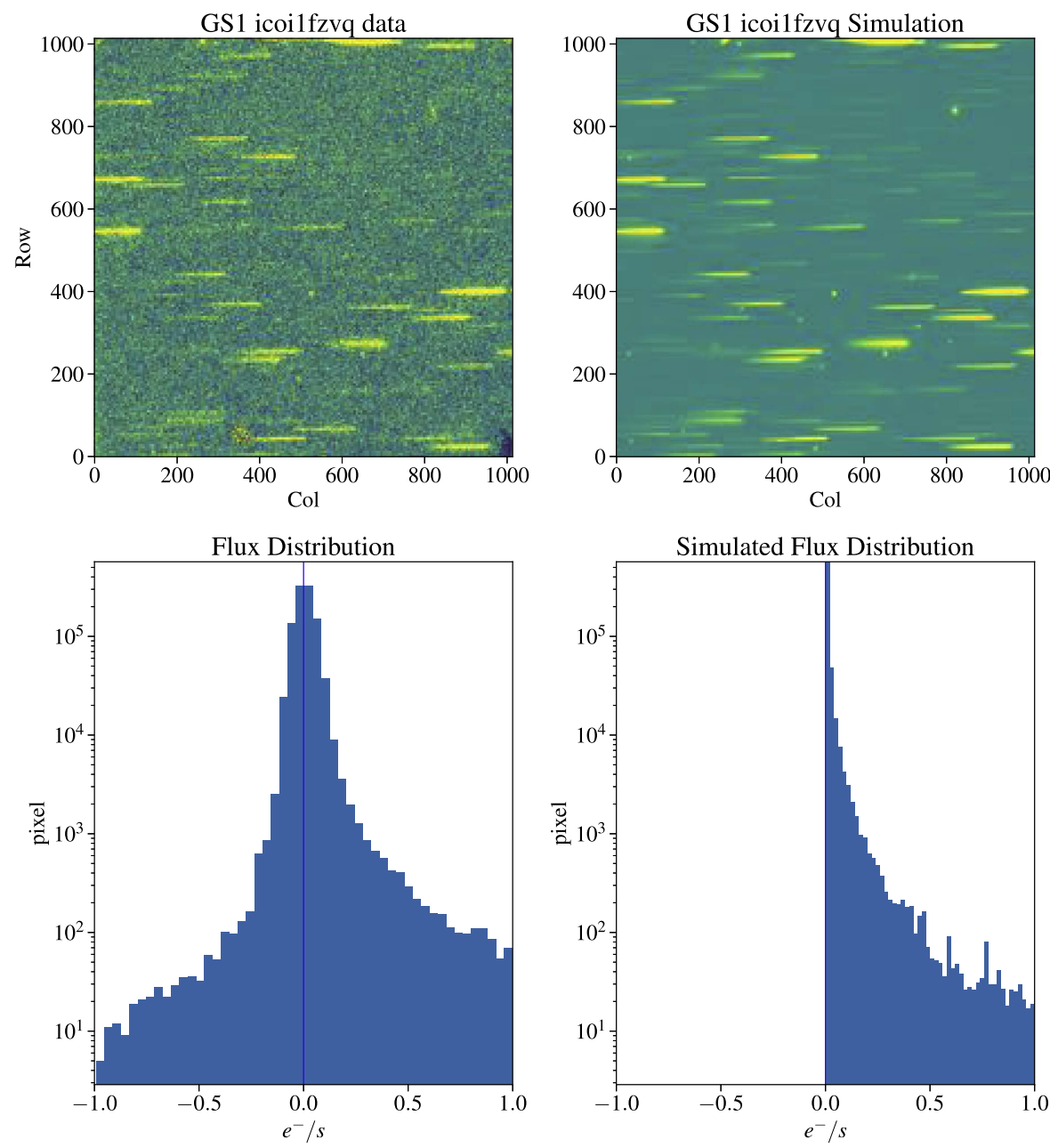

Figure 7. Same as Figure 5 but for the GS1 field.
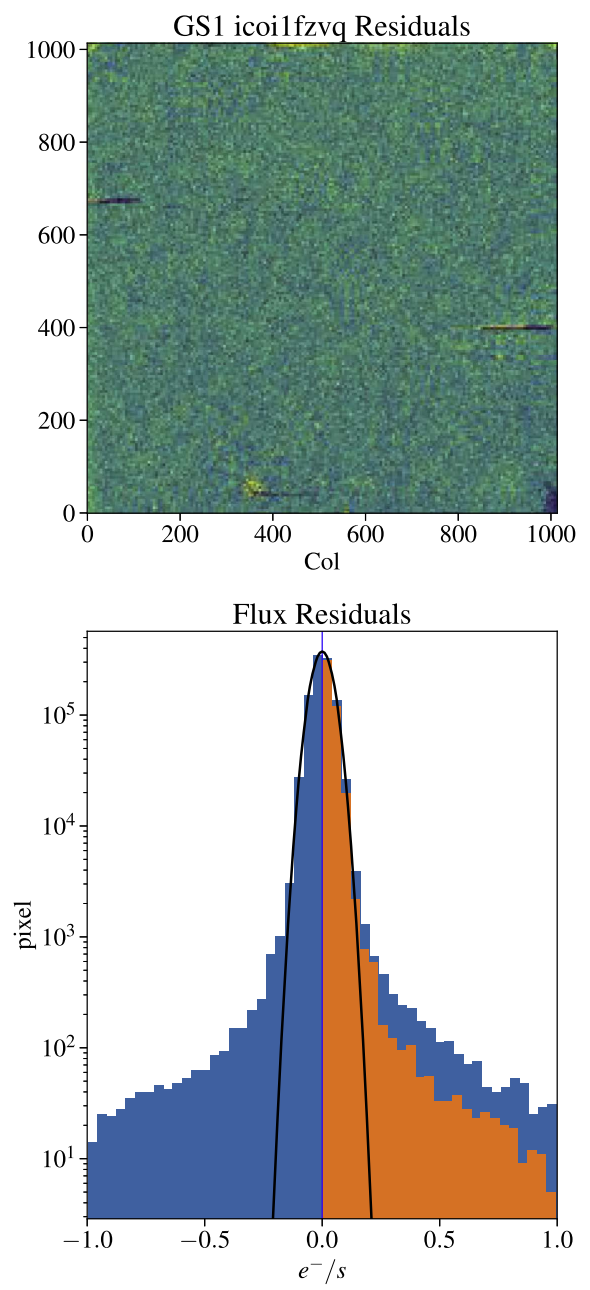

picked up in the hot catalog. We also visually identified objects that were split by the catalog process and merged them in the final catalog (recalculating the shape parameters from the final, combined objects). Faint sources near bright objects were assumed to be part of the bright object, although we performed intensive visual inspection of our catalogs when deriving our extraction parameters to ensure that we did not under- or oversplit bright, extended objects. We compared this catalog to the known positions of high-redshift galaxies using a master catalog of known objects from Finkelstein et al. (2015) and Bouwens et al. (2015). Some of the fainter objects were not present in our catalog. These objects are desirable, as detecting Ly $\alpha$ emission from such sources is one of the main goals of this survey. We thus ran a "super-hot" catalog with DETECT_THRESH $=0.35$ and DETECT_MINAREA $=14$, which identified missing sources from those catalogs and added them to our final catalog.

\subsubsection{Astrometric Correction}

The astrometric reference frame for the FIGS fields were provided by the large 30 mas scale mosaics of the fields discussed in Section 3.2.1. We started by using the SWarp program to generate deep images of the field, properly oriented and with the native WFC3 pixel scale for each of the FIGS visits. Individual FIGS F105W direct images, which were taken during each of the FIGS visits to provide an astrometric reference frame for the G102 exposures, were then astrometrically registered to the deep, rotated mosaics. While we initially used the Astrodrizzle task Tweakreg to perform this task, we found that we could not properly control which objects in the field were used to compute the $\mathrm{x}$ and $\mathrm{y}$ offsets between our FIGS F105W images and our master mosaic images. Unfortunately, the master mosaics were generated with data that are several years old, and each of the FIGS field contains bright stars with a significant amount of proper motion ( $<3$ mas $\pm 5 \mathrm{yr}^{-1}$, on average; Windhorst et al. 2011). It was therefore preferable to use many faint sources (galaxy or stars) to astrometrically register these images to our master mosaics, as was done in Pirzkal et al. (2005). An iterative version of the FOCAS (Valdes et al. 1995) triangulation algorithm was implemented to register the geometrically distorted FIGS F105W FLT (HST pipeline calibrated exposure) images to the deep, rectified FIGS mosaics. During each iteration, between 40 and 1000 sources were matched and averaged R.A. and decl. shifts were computed as well as any needed rotation. These were applied as a correction to the FIGS F105W image and the G102 images taken during the same HST visit. We found this approach to be accurate with residuals on the order of 0.2 WFC3 IR pixels (25 mas), or about two times better than those we were previously able to achieve. Figure 4 shows histograms of the computed R.A. and decl. offsets, as well as the rotations needed to match the FIGS data to the 

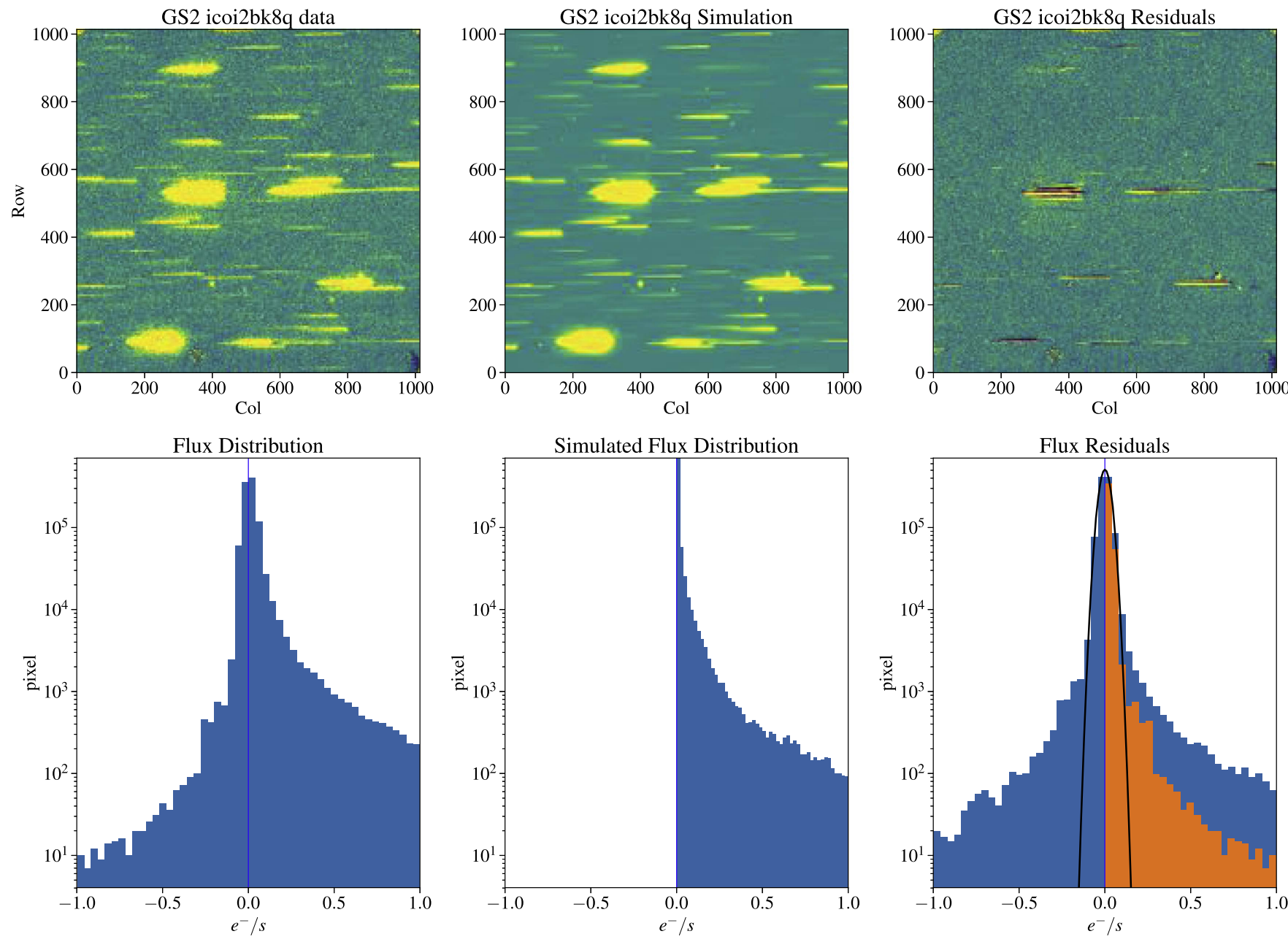

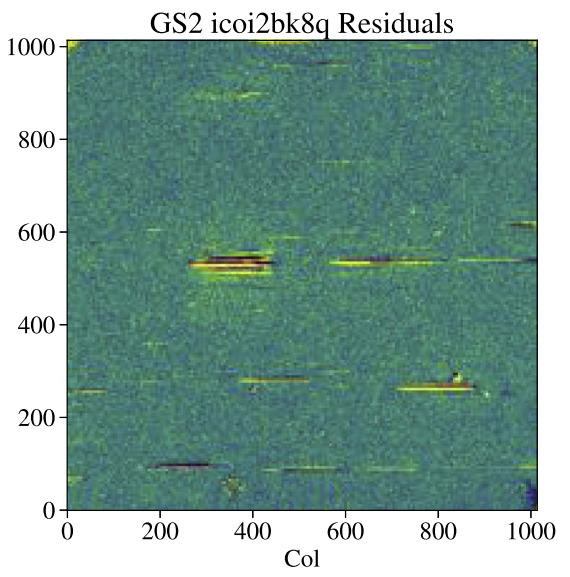

Figure 8. Same as Figure 5 but for the GS2 field.

reference mosaics, described in Section 3.2.1. As this figure demonstrates, the computed corrections vary from field to field as different guide stars are used for each field and orientation.

\subsubsection{Simulations and Simulation-based Extraction}

When analyzing slitless data, one must necessarily have some knowledge of where each source is expected to be in the field, and also have a good understanding and calibration of the WFC3 G102 grism (Pirzkal et al. 2016). Since spectra of different objects overlap in our observations, we must be able to estimate where light for every single source in the field will be dispersed to for all five different spectral orders of the G102 grism. While we are only interested in extracting data from the first dispersed order, a complete tally of the contamination from other objects and spectral orders is required. We used the publicly available WFC3 G102 grism calibration file (Pirzkal et al. 2016; Pirzkal \& Ryan 2017a) with new custom software to simulate every single FIGS grism observations. These simulations were based on the broadband continuum SED of objects in the field. While bright emission lines in higher spectral orders are not simulated at this stage, the use of data taken at multiple position angles is sufficient to exclude such lines or other artifacts, such as bonafide emission lines, in observed first-order spectra. The software dispersed every object pixel in the master mosaics onto the reference frame of the FIGS observations, using the multiple broadband mosaics to assign a spectral energy distribution to each pixel. For each pixel, the broadband fluxes are interpolated to form a smooth SED. This process is similar to how the aXe (Pirzkal et al. 2001; Pasquali et al. 2006; Kümmel et al. 2009) software package models observations. However, it allowed us to compute the dispersion solution only once and to store this information for later use when extracting data or estimating the contamination level in a spectrum. The SBE approach produces large tables containing, for each grism pixel in every database, a list of what object pixel contributed what amount of flux and at what wavelength. These large data cubes can be used for a variety of tasks, such as generating simulated dispersed images for individual objects or all objects. These data cubes can also be used to determine which pixel in the real observations need to be extracted and co-added to produce "rectified" 2D images, where the $x$-axis is now a linear function of wavelength. This approach is also well suited to generate forward modeling models of star-forming regions, as will be presented in a forthcoming paper N. Pirzkal et al. (2017, in preparation). One additional advantage of the SBE approach is that we are able to compare simulations to observations early in the analysis process. When the astrometry and input catalogs are sufficiently accurate, the simulations and the observations should be very similar. Thus, it is a confirmation of the accuracy of any future individual object contamination estimates, as well as any 


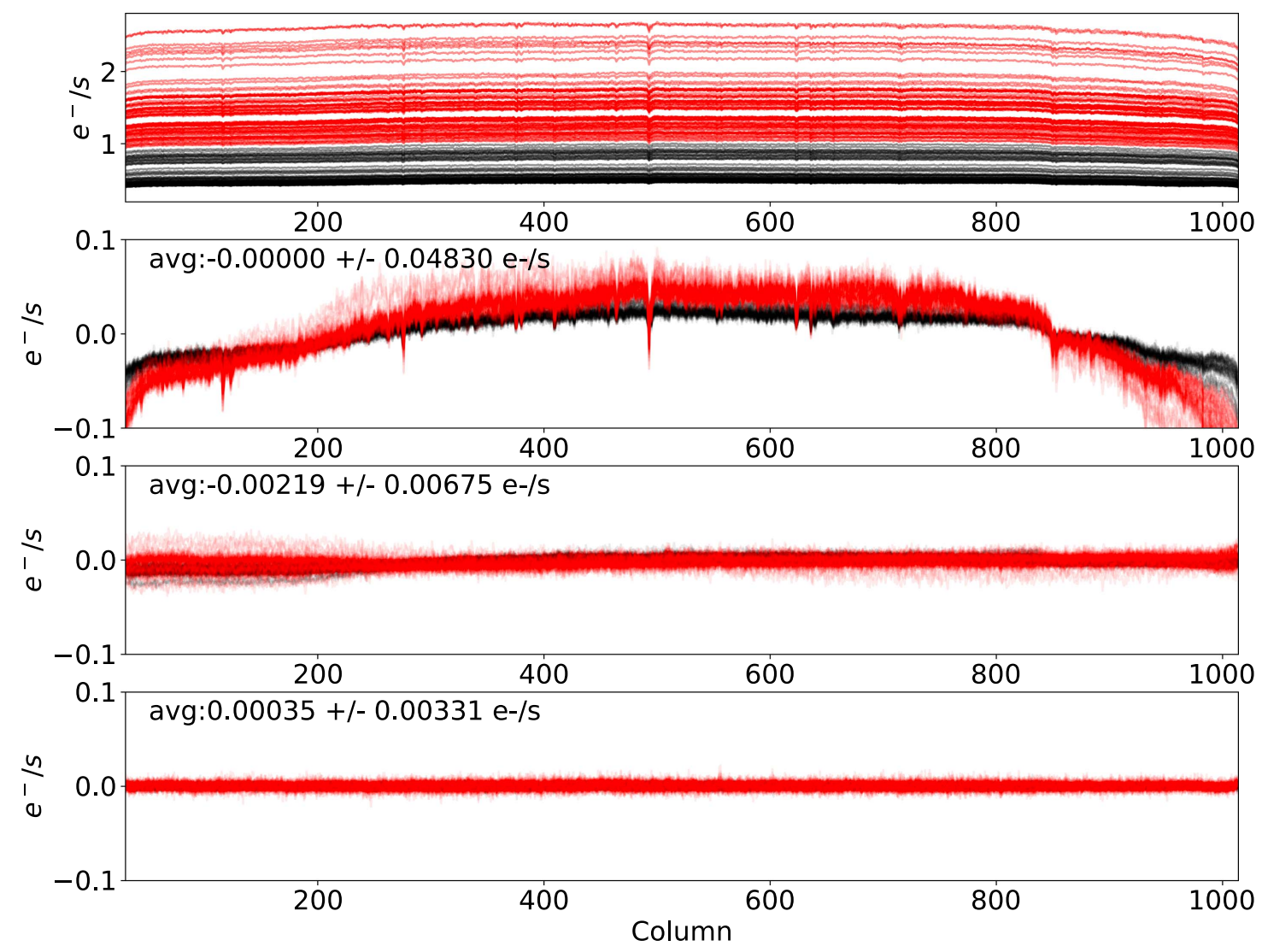

Figure 9. Median column values, as a function of column, of all the observations taken for the FIGS GS1 field. The top panel shows the original background levels of the observations. Observations with a large amount of He I light are plotted in red. The second panel shows the mean subtracted background residuals if a constant dispersed background is used, and shows how observations with a large amount of He I have a different background structure. The third panel shows the result of implementing the two components and varying the He I level background subtraction described in Section 3.2.6. Finally, the fourth panel shows the residuals when an additional smooth component is fitted and subtracted. As we show here, the FIGS background subtraction brings the background level of our grism observations to within $0.003 e^{-} \mathrm{s}^{-1}$ of zero.

future extracted "rectified" 2D image of individual objects in the field. In Figures 5-8, we compare a single FIGS observation to its FIGS simulation. The lack of significant shift in the $\mathrm{x}$ or $\mathrm{y}$ direction (except for the brighter stars with detectable proper motion) indicates a good astrometric solution. The quality of the continuum level subtraction is an indication that our estimate of the SED of each pixel was also accurate. Regions with poor subtractions are caused by saturation of bright objects, persistence effects, and proper motions of some of the bright stars in the fields.

\subsubsection{Persistence Flagging}

Persistence (Long et al. 2013) affects many WFC3 IR exposures. In the case of FIGS data, it can be caused by previous FIGS exposures themselves, or by other HST programs executed before a given FIGS exposure. We used the $H S T$ archive to identify every exposure obtained within 36 hr of a FIGS exposure and flagged any pixels that were saturated within that period. Typically, at most, a few hundred pixels were affected in a particular image. Flagging these pixels was essential since persistence could mimic faint emission lines.

\subsubsection{Background Subtraction}

The dispersed background light must be subtracted from slitless observations, so that any under or over subtraction of the background sets a limit on our ability to detect faint continuum or emission lines. The dispersed background not only has spatial structure imposed by the multiple overlapping spectra orders, but can also vary substantially during the course of an observation. This is because the bright Earth limb contributes to the overall background light in the form of He I emission from the Earth's upper atmosphere. At the beginning and end of an observation, $H S T$ is more likely to be pointing close to the Earth's limb, and to be affected by He I emission. Unfortunately, the default HST WFC3 data calibration pipeline (CALWF3) cannot handle images with varying backgrounds properly when up-the-ramp fitting (UTRF) is used (Robberto 2007). CALWF3 uses UTRF of multiple non-destructive reads to produce images with a lower effective read-noise $\left(12 e^{-}\right.$versus $\left.20 e^{-}\right)$, which is free of cosmicray impacts. To deal with this significant issue, we assumed that the dispersed grism background is a linear combination of two separate contributions: the regular and constant dispersed Zodiacal light, and a time varying dispersed He I background. These two components were previously modeled and are described in Brammer et al. (2015). We then estimated the amount of He I light separately in each of the multiple reads taken during the exposure. This He I estimate was then subtracted from each of the reads, thereby removing the varying component from the observation, UTRF was then used to produce a set of intermediate images. The Zodiacal light was then subtracted from these images to produce a final set of images. This method is described in detail in Pirzkal \& Ryan 


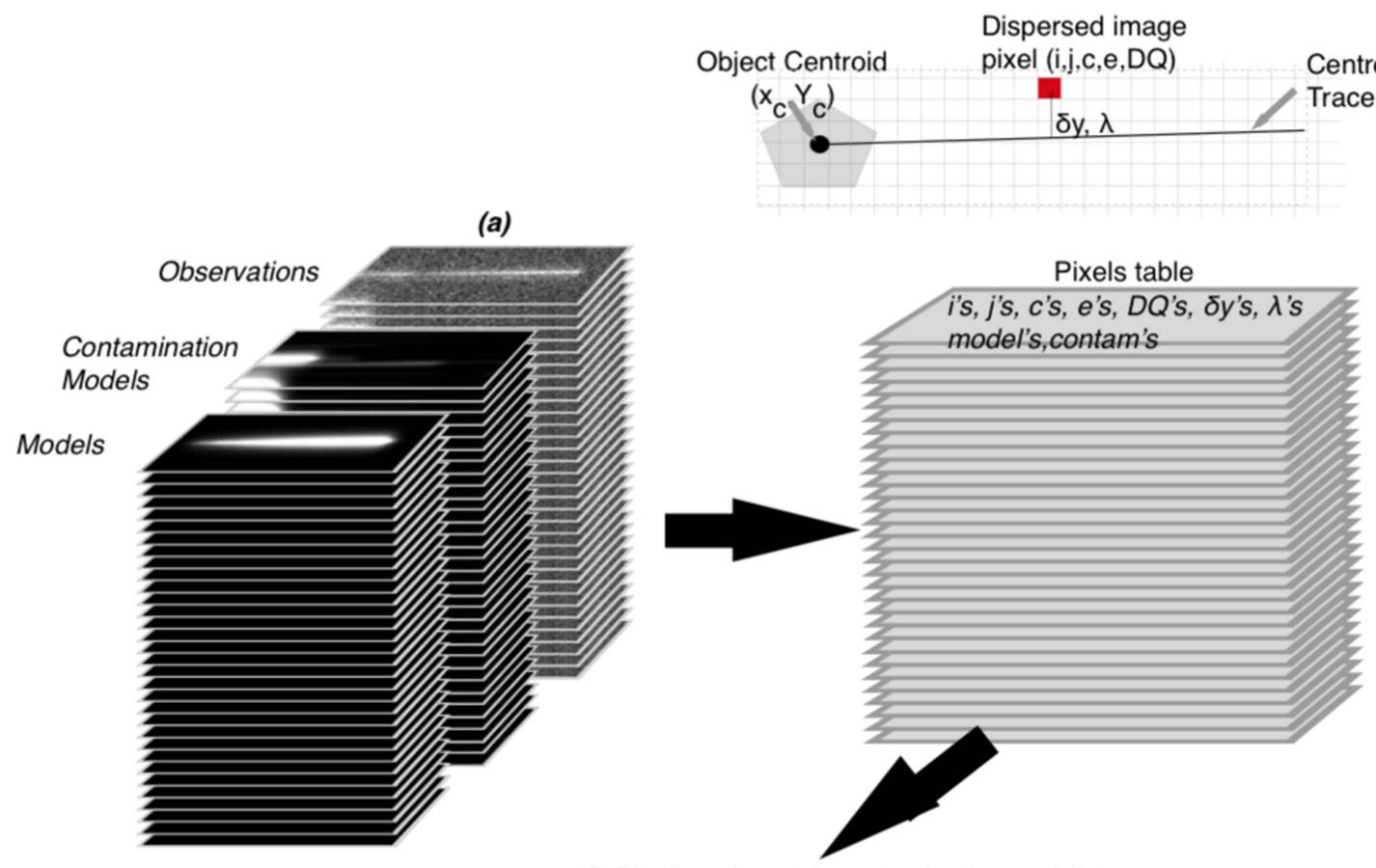

(b)

$2 D$ Binning of model, contamination and data

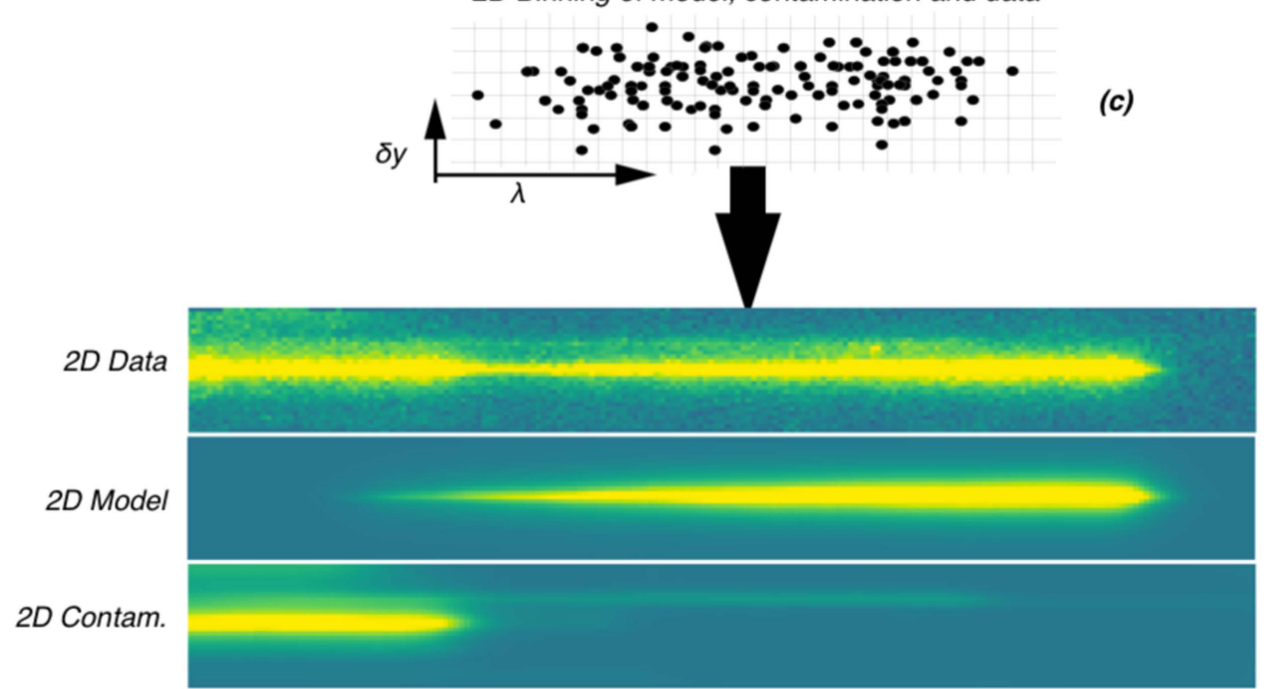

(d)

Figure 10. Extraction process described in Section 3.3.1. Stacks of observed data, and models (panel (a)) combined with the known field-dependent G102 grism dispersion are used to generate the pixel tables (panel (b)). The pixel table contains, for each pixel, the detector coordinates ((i), (j)), flux (c), flux error (e), data quality flag (DQ), distance of the center of the pixel to the trace $(\delta y)$, and assigned wavelength $(\lambda)$, The latter can be used to map the observations, model estimates as well as contamination, in a wavelength vs. cross-dispersion distance ( $\lambda$ vs. $\delta y$ ) space (panel (c)). When mapped into this two-dimensional space, the flux (c) can be binned two-dimensionally in lambda and $\delta y$. Flux values in each bin are combined using a $3 \sigma$ clipping weighted mean, using the original error estimates (e) of the original G102 fluxes (c). The standard deviation of this mean is computed and serves as a robust and empiracal estimate of the error in each bin. This binning process results in combined 2D images of the flux, error, model, and contamination estimates that are wavelength calibrated (d) and rectified in the cross-dispersion direction.

(2017b). While observations typically have background levels of $\approx 0.5-1.4 e^{-} \mathrm{s}^{-1}$ with spatial variations of $\approx 0.05 e^{-} \mathrm{s}^{-1}$, this method yields images with a background residual of $0.002 \pm$ $0.005 e^{-} \mathrm{s}^{-1}$. This was further improved by computing the median of an observation in the row direction, and then smoothing and subtracting it from the data. The first step in this further refinement was to mask pixels affected by a level of persistence that is 0.6 times higher than the error estimate, and then to mask pixels affected by dispersed spectra with a count rate larger than $0.01 e^{-} \mathrm{s}^{-1}$ (as determined in our simulated images discussed in Section 3.2.4). This final step further reduced the background residuals to a level of $\pm 0.003 e^{-} \mathrm{s}^{-1}$.
Figure 9 shows the result of this process for the FIGS GS1 observations.

\subsection{Extraction}

\subsection{1. $2 D$ Extraction}

Each object present in the SeXtractor segmentation file described in Section 3.2.2 was extracted. First, for each available FLT file, the dispersed trace corresponding to the centroid coordinates listed in the FIGS catalog was computed. Then, pixels in the FLT data (avoiding those flagged as bad in the FLT Data Quality extension) were assigned a wavelength $(\lambda)$ 

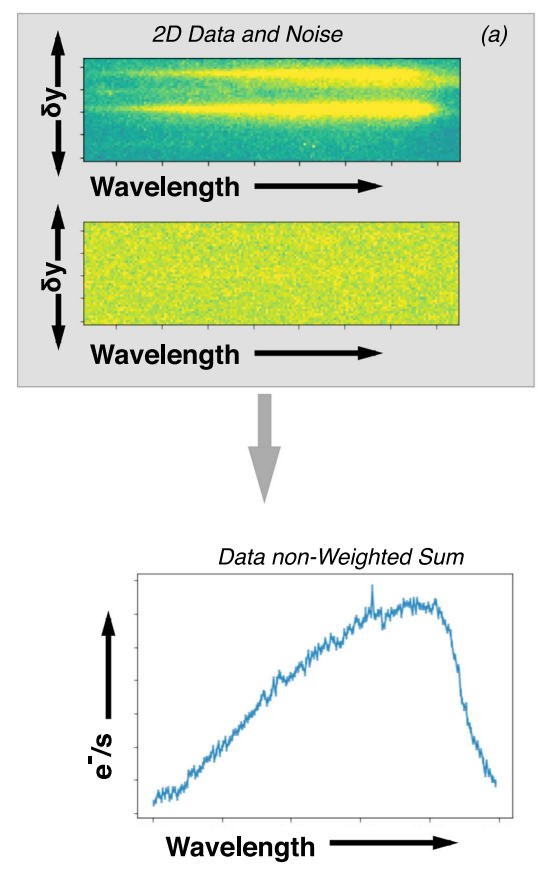
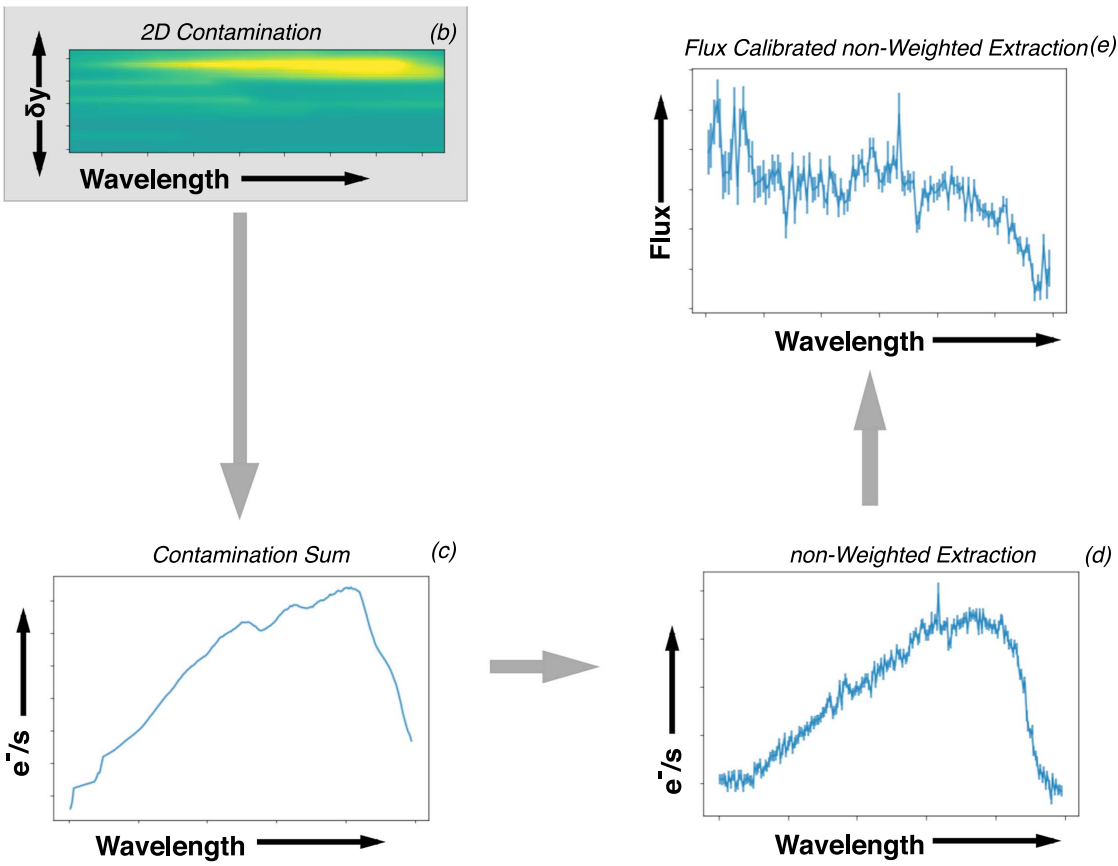

Figure 11. FIGS non-weighted extraction process: the data and error 2D data (a) are summed and errors are propagated to produce a 1D spectrum of the source. The same process is used with the $2 \mathrm{D}$ contamination estimate (b) for this object to produce a 1D spectral estimate of the contamination (c). The 1D contamination estimate is subtracted from the 1D source spectrum to produce a contamination-free spectrum (d). The final flux-calibrated 1D spectrum is then generated by applying the grism sensitivity (e).

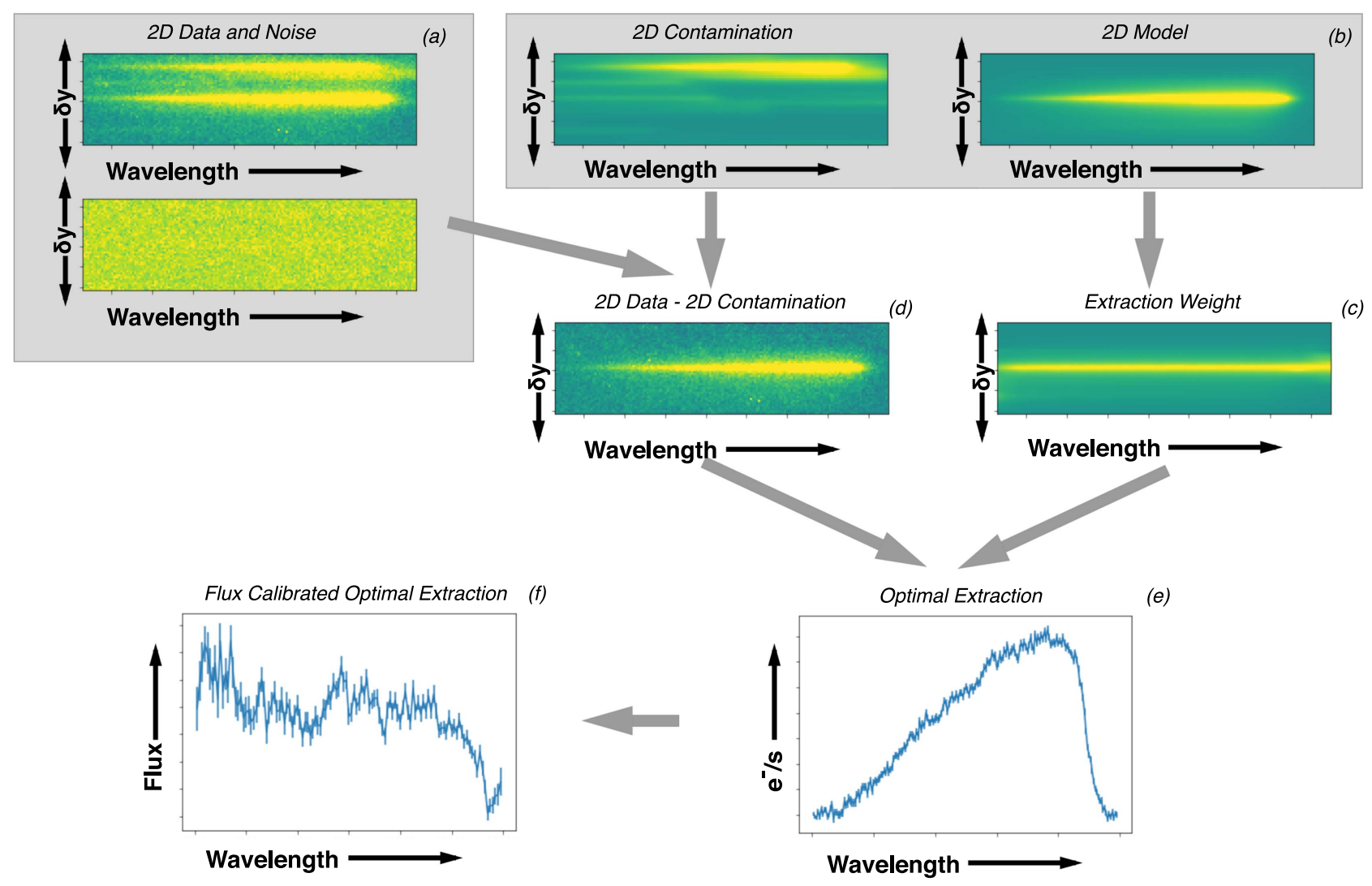

Figure 12. FIGS Optimal Extraction process: the $2 \mathrm{D}$ contamination (b) is first subtracted from the $2 \mathrm{D}$ data (a) to produce a contamination-free $2 \mathrm{D}$ estimate (d). The 2D model of the dispersed spectrum of the source (b) is used to create a normalized extraction weight image (c). The optimal extraction algorithm from Horne (1986) combined data, error, and extraction weight to produce a $1 \mathrm{D}$ estimate of the spectrum of the source (e). The final flux-calibrated $1 \mathrm{D}$ spectrum is then generated by applying the grism sensitivity (f). 


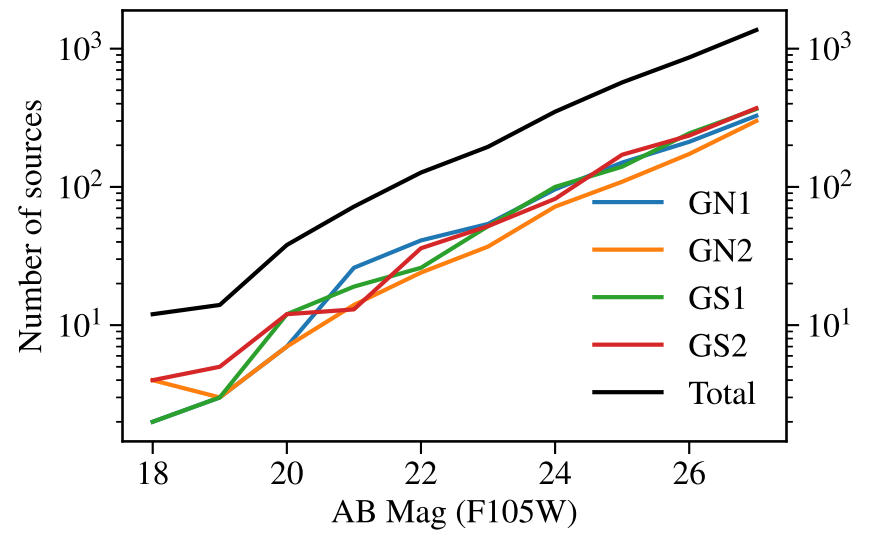

Figure 13. Number of source spectra as a function of source $m_{F 105 W}$ magnitude for each FIGS field and for the total FIGS sample (black).

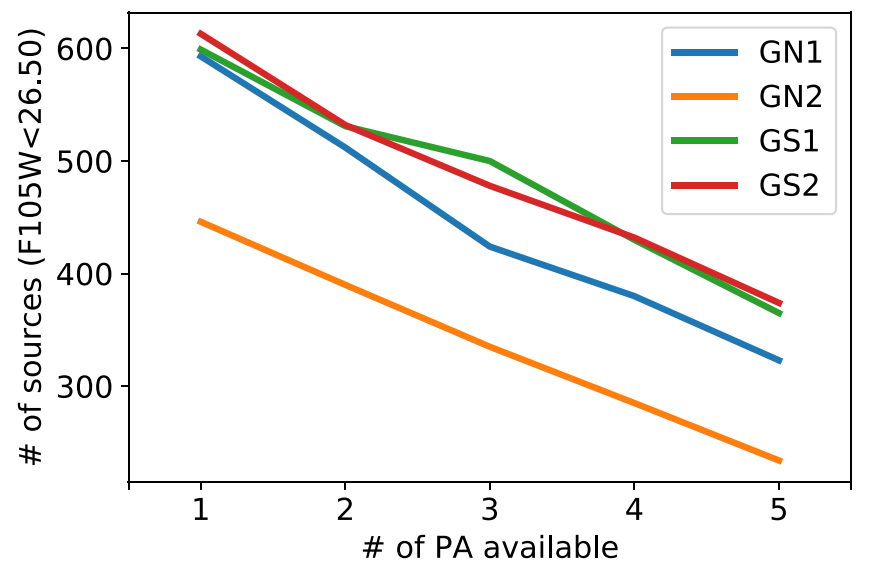

Figure 14. Number of objects with $m_{F 105 W}<26.5$ magnitude, observed in 1 , $2,3,4$, or 5 position angles. Approximately $55 \%$ of all of the sources were observed to the full depth of 40 orbits.

and the cross-dispersion distance $(\delta y)$ between the dispersed centroid trace and the center of that pixel was computed. We also used the simulated data cubes discussed in Section 3.2.4 to determine the amount of flux in that pixel contributed by the spectra of other sources in the field. These three quantities supplement the already known observed count rate, error estimate, and data quality flag of that pixel. This is very similar to what the aXe extraction software does (Kümmel et al. 2009). However, our approach differs in that, unlike aXe, this information was not used to produce individual FLT extraction of the spectrum of each object. Instead, the information obtained using all of the FLT data obtained at the same PA on the sky (i.e., 16 or 32 of them) were combined. A two-dimensional, wavelength rectified image was then generated by binning those data in $\lambda$ and $\delta y$ space. The bins were chosen to be $25 \AA$ in the wavelength direction and one WFC3 pixel in the $\delta \mathrm{y}$ direction $(0 . \prime 129)$, as these are close to the native properties of the instrument. Since we had data from multiple, dithered FLT files to bin, the number of the available sample in each bin was sufficient to both derive a robust ( $3 \sigma$ clipping mean) estimate of the count rate in each bin, as well as to derive an accurate standard deviation of the mean for each of the two-dimensional bins. The identical process was followed to generate $2 \mathrm{D}$ rectified images of the simulated spectrum of each source, as well as of the simulated contamination estimate for each source. This process is illustrated in Figure 10.

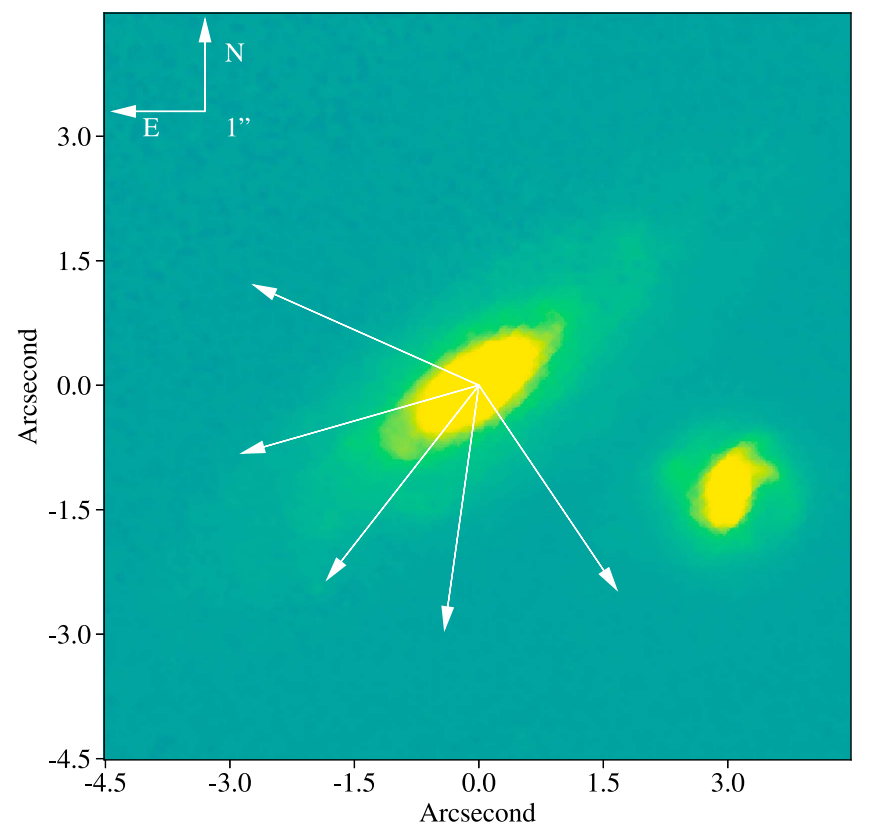

Figure 15. Extended, asymmetrical galaxy in the GN1 field, and the five directions along which spectra were obtained.

\subsubsection{D Extraction}

One-dimensional extractions were created from the 2D-extractions using two methods: non-weighted extraction and optimal extraction. These methods are shown graphically in Figures 11 and 12. In the first instance, rows of the 2D rectified spectra (Figure 11(a)) are simply co-added. The SeXtractor segmentation footprint determines which rows should be included in this summing operation. The 2D model of the spectral contamination (Figure 11(b)) is also co-added to create a $1 \mathrm{D}$ spectral estimate of the contamination. The latter is subtracted from the 1D spectrum of the source (Figure 11(c)) and a final 1D spectrum is generated by applying the known grism sensitivity (Figure 11(e)).

The FIGS optimal extraction follows a non-iterative version of the algorithm described in Horne (1986): we used the simulated version of the 2D dispersed spectrum of the source to determine the expected profile of the spectrum as a function of wavelength (Figure 12(b)). This profile was normalized to unity in the cross-dispersion direction and used as the extraction weight (Figure 12(c)). This extraction weight was then used in combination with the 2D contamination subtracted 2D data (Figure 12(d)), to produce an optimally extracted 1D spectrum (Figure 12(f)). The optimal extraction has the advantage of producing higher $\mathrm{S} / \mathrm{N}$ spectra with improved flux calibration, but only when the extraction weights (derived from the imaging data) are accurate. This is not always the case, as in the example of stars with proper motion. In such cases, the extraction weights are misaligned and cause spectral artifacts in the extracted data. Checking the consistency between the co-added and optimally extracted spectra is always recommended.

\section{FIGS Spectra}

\subsection{Depth of Complete Survey}

The number of FIGS spectra, up to five per source, as a function of broadband $m_{F 105 W}$ magnitude is shown in the right 

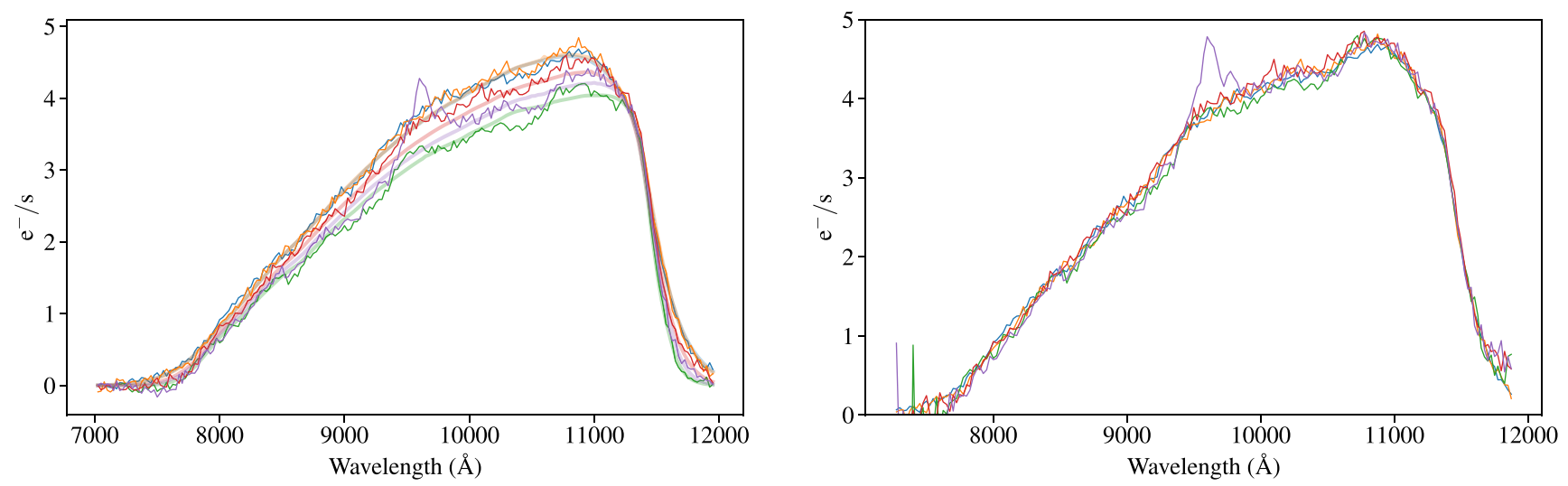

Figure 16. Left: non-flux-calibrated 1D spectra of the large galaxy shown in Figure 15 as well as the FIGS model of the same galaxy. The large asymmetry of the galaxy results in a significantly different amount of smoothing, which results in wider, as well as overall lower amplitude spectra. Right: same spectra as shown in the left panel, but an object-specific correction based on the FIGS extraction of the simulation of this object was applied.
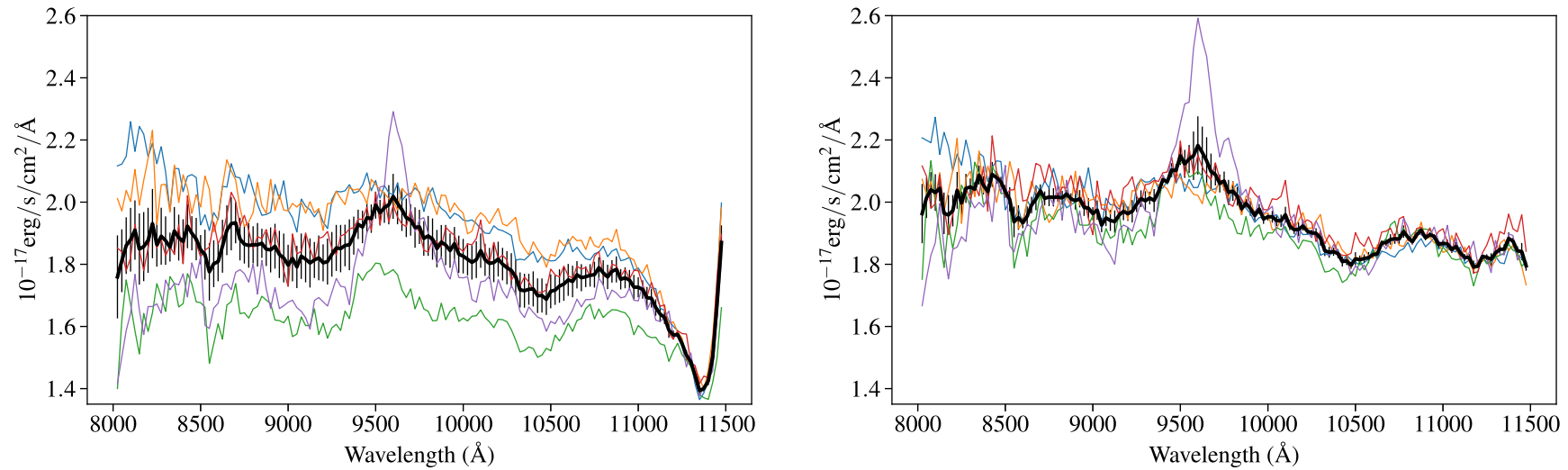

Figure 17. Left: same as the left panel of Figure 16, but after applying the default G102 spectral sensitivity function. We also show the result of combining these spectra (black line with error bars). Right: flux-calibrated spectra of this source after applying an object-specific G102 spectral sensitivity function. The resulting combined spectrum (shown in black) is significantly less noisy, and does not suffer from the edge effects of the combined spectrum shown in the left panel.The broad spectral feature at $9500 \AA$, which could be the result of some extended $\mathrm{H} \alpha$ emission, is readily visible in the final combined spectrum.

panel of Figure 13. The total number of sources extracted in each of the FIGS fields were determined by the depth of the available imaging data and the size of the catalog for each field, as well as the specific position angles. The total number of extracted sources for the GN1, GN2, GS1, and GS2 fields are 1913, 1003, 3106, and 2623, respectively. The increased number of extracted spectra in the GS1 and GS2 is due to the deeper images and larger object catalogs available for these fields. The number of sources brighter than $m_{F 105 W}=26.5 \mathrm{mag}$ that were extracted are 595, 453, 603, and 619 for GN1, GN2, GS1, and GS2, respectively, The total number of extracted spectra for all four fields brighter than $m_{F 105 W}=26.5 \mathrm{mag}$ is 2270 .

Not every source was observed to the full depth corresponding to five position angles, and Figure 14 shows the number of sources brighter than $m_{F 105 W}=26.5$ mag that were observed in at least $n$ position angles. Approximately 57\% of the 2270 sources brighter than $m_{F 105 W}=26.5$ mag were observed to the full depth of the survey, or $323,234,365$, and 374 sources in GN1, GN2, GS1, and GS2, respectively.

\subsection{Combined Spectra}

Each source in the FIGS survey was observed up to five times, producing five distinct spectra. Due to the properties of slitless spectroscopy, spectra obtained for even moderately

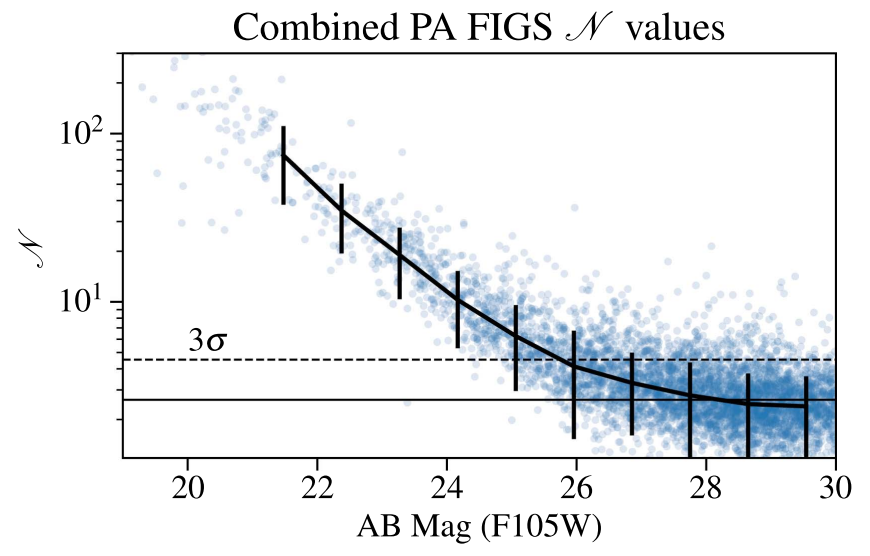

Figure 18. Distribution of $\mathcal{N}$ values for combined PA-depth FIGS spectra (blue). The mean value of $\mathcal{N}$ is shown in black with error bars and approaches the value of $\mathcal{N} \approx 2.6$ (lower horizontal black line) for sources that are several magnitudes fainter then our detection limit. The $3 \sigma$ continuum level at a limit of $\mathcal{N} \approx 4.5$ is also shown (dashed line).

extended object are subject to a different amount of smoothing. Thus, the resolution is set by the image of the object itself.

The observed spectra are the convolution of the light profile of the object with its spectrum, and large differences in this light profile between different PA (for example, in the cases of 

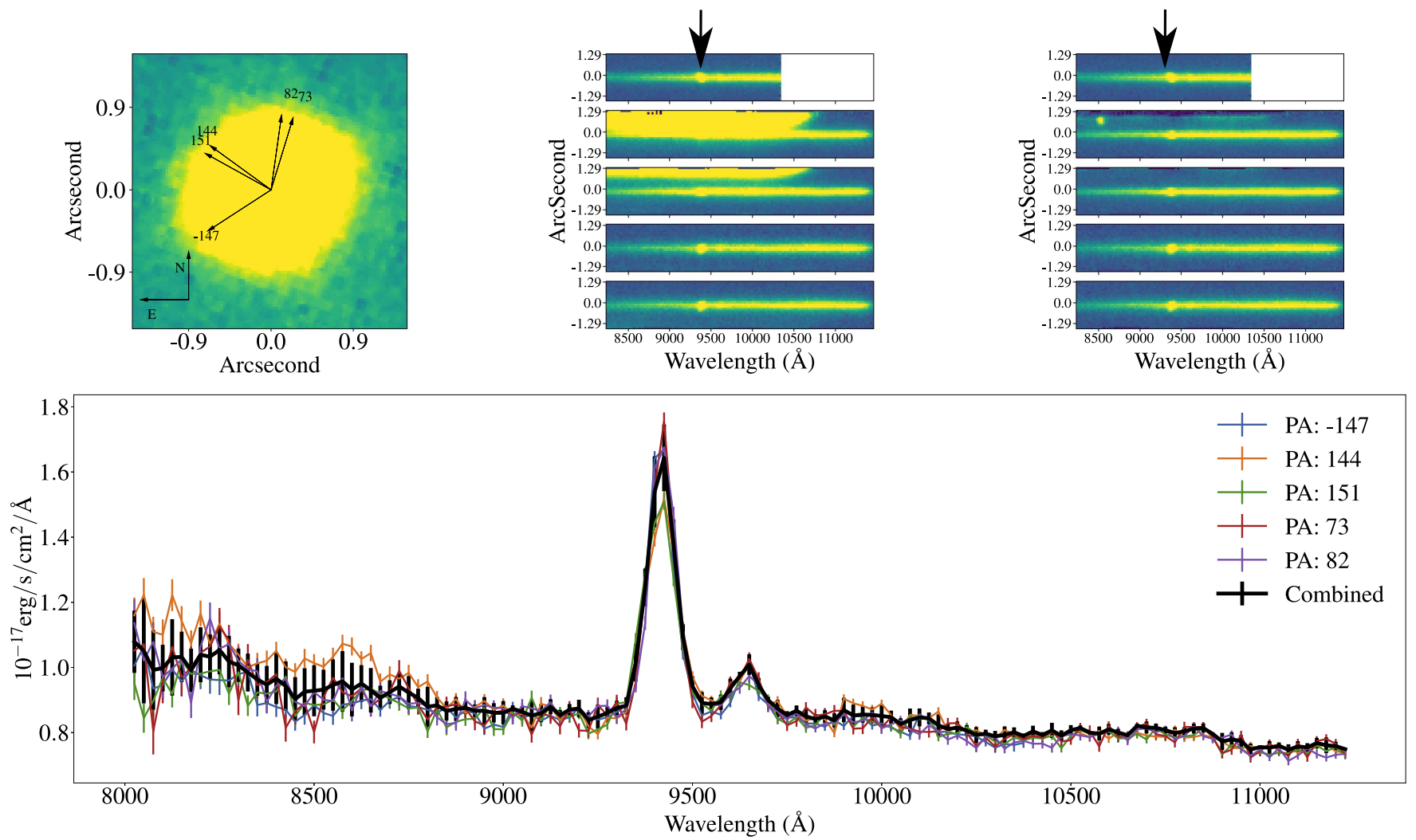

Figure 19. $m_{F 105 W}=20.23 \mathrm{mag}$ source in the GS1 field at a redshift of $z=0.43$ with prominent $\mathrm{H} \alpha$ and $[\mathrm{S}$ II] lines $(\mathcal{N}=304$.). The $\mathrm{H} \alpha$ line flux is $5 \times 10^{-16} \mathrm{erg} \mathrm{s}^{-1} \mathrm{~cm}^{-2}$. The top left panel shows the F105W image and the orientation of the five PAs used to observed this source. The top middle panel shows the $2 \mathrm{D}$ rectified spectra. The top right panel shows the contamination-corrected 2D rectified spectra with black arrows pointing to the emission lines. The bottom panel shows the individual 1D spectra (color) as well as the combined spectrum (black) lines.

elliptical or irregular galaxies) will result in spectra that disagree strongly near the edge of the bandpass of the grism. They will also have continuum fluxes that are in disagreement, as the spectrum is smoothed by different amounts. This effect is illustrated in Figures 15-17, where we show the example of a large galaxy. We derived an object-specific spectral response for each source by dividing the extracted 1D data by the extracted 1D simulated data, and by the spectral energy distribution used to generate the FIGS simulations, which were generated from the available FIGS broadband photometry. The result is a normalized spectrum, which can be scaled back to the observed F105W photometry. These steps ensure that the 1D spectra of extended sources are accurately flux calibrated and avoid the issue of having a point-source sensitivity function applied to an extended object.

The FIGS spectra were flux calibrated using object-specific sensitivity functions and then combined. For each wavelength bin, the inverse variance of the single-PA spectra were used as weights to compute the weighted mean and standard deviation of the weighted mean. An iterative $3 \sigma$ rejection was used to remove outlier single-PA spectral bins.

\subsection{Net Significance}

The information content of a spectrum can be better described by the net significance $(\mathcal{N})$, which was introduced in Pirzkal et al. (2004). $\mathcal{N}$ is the maximum cumulative $\mathrm{S} / \mathrm{N}$ of a spectrum. We compute it as follows, for each spectrum.
1. Divide the $\approx 140$ flux values $F_{i}$ by their respective error estimates $e_{i}$ to produce $140 \mathrm{~S} / \mathrm{N}$ estimates $S N_{i}$.

2. Reorder the original flux and error arrays according to the descending order of the $\mathrm{S} / \mathrm{N}$ estimates to produce $F_{i}^{\prime}$ and $e_{i}^{\prime}$.

3. Compute $\mathcal{N}=\sum_{i=0}^{n} F_{i}^{\prime} / \sqrt{\sum_{i=0}^{n}\left(e_{i}^{\prime}\right)^{2}}$ for increasing values of $n$, until the maximum value of $\mathcal{N}$ is reached.

Figure 18 shows a plot of the computed $\mathcal{N}$ for the combined five PA-depth FIGS spectra. Sources fainter than $m_{F 105 W}=28$ are below our G102 grism sensitivity and Figure 18 shows that $\mathcal{N}$ approaches its limiting value of 2.6. A simulated spectrum with a continuum level of $3 \sigma$ per bin, is expected to have $\mathcal{N} \approx 4.5$. There are several factors that can of course affect a value of $\mathcal{N}$ and a contamination or detector artifact that is unaccounted for can result in an artificially high value of $\mathcal{N}$. Similarly, a small error in the background subtraction can raise or lower the level of any continuum signal, affecting the value of $\mathcal{N}$. As a whole, the $\mathcal{N}$ distribution shown in Figure 18 indicates that, on average, the $3 \sigma$ detection limit of the FIGS survey corresponds approximately to a $m_{F 105 W}=26$ continuum source.

\subsection{Examples}

We show two examples of extracted FIGS spectra in Figures 19 and 20, in addition to Figure 17, where we showed the spectra of a large galaxy at $z_{\text {phot }} \approx 0.42$. The first shows a relatively bright galaxy with a prominent $\mathrm{H} \alpha$ line, as well as an [S II] line. This Figure shows the level of consistency one can expect between observations taken at different PA, even though 

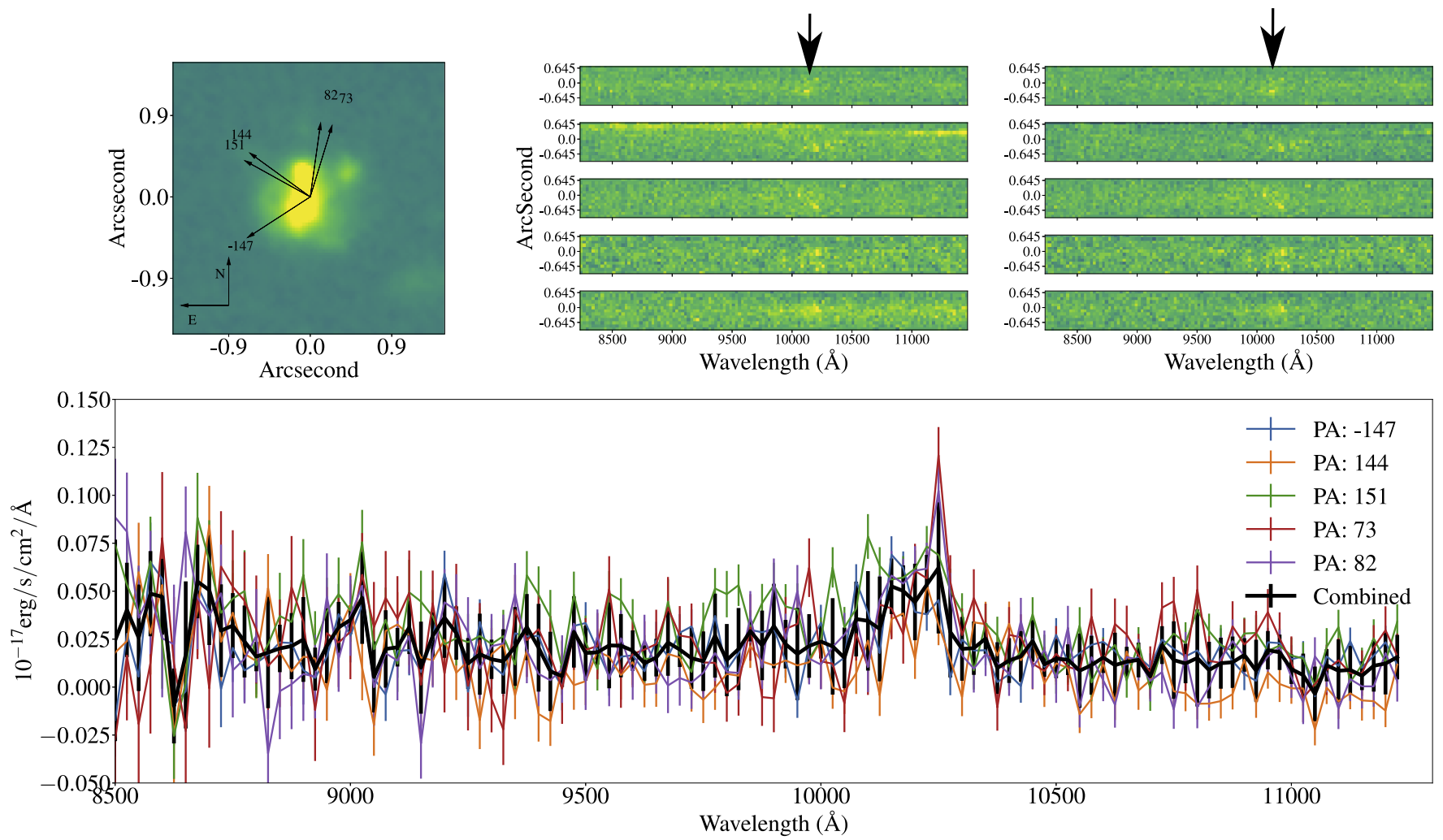

Figure 20. $m_{F 105 W}=24.1 \mathrm{mag}$ source in the GS1 field at a redshift of $z=1.03$ with an extended [O III] emission line $(\mathcal{N}=6.95)$. The flux of this emission line is $3 \times 10^{-17} \mathrm{erg} \mathrm{s}^{-1} \mathrm{~cm}^{-2}$. The top left panel shows the F105W image and the orientation of the five PAs used to observed this source. The top middle panel shows the $2 \mathrm{D}$ rectified spectra. The top right panel shows the contamination-corrected $2 \mathrm{D}$ rectified spectra with black arrows pointing to the emission line. The bottom panel shows the individual 1D spectra (color) as well as the combined spectrum (black) lines.

the amount of contamination varies greatly. The second example shows a much fainter galaxy with unresolved [O III] emission, which can be seen to have structure in the 2D spectra, and correlates with the clumpy morphology of this galaxy. An example of a very faint high-redshift FIGS galaxy at a redshift of 7.51 with a $1.06 \times 10^{-17} \mathrm{erg} \mathrm{s}^{-1} \mathrm{~cm}^{-2}$ Ly $\alpha$ emission and possibly NV emission, suggesting that this source might contain an active galactic nucleus, can be found in Figure 1 of Tilvi et al. (2016).

\section{Conclusion}

We have described the survey design, methodology, data reduction, and data analysis of FIGS, a deep WFC3/IR slitless spectroscopic survey using the G102 grism filter. We have extracted spectra of 8645 individual sources, 1296 of which are of objects brighter than $m_{F 105 W}=26.5$ mag that were observed to the full survey depth of 40 orbits per field.

The reduced and calibrated FIGS spectra are available via the MAST data archive (doi:10.17909/T9010Z) and include the single-PA 2D data stamps, the single-PA extracted spectra using optimal extraction, and finally, the combined 5-PA versions of the 1D spectra.

We expect these deep, multiple PA slitless observations to pave the way for new deep slitless observations from new space-based missions. First, most instruments with the James Webb Space Telescope (JWST) will be grism-capable; in fact, several will have multiple grism elements that disperse the light in orthogonal directions to mitigate contamination from overlapping spectral traces. Second, the Wide-Field Infrared
Survey Telescope (WFIRST), which is expected to survey $\gtrsim 2000 \mathrm{deg}^{2}$ and collect $\gtrsim 10^{7}$ redshifts at $1 \lesssim z \lesssim 3$ as a means of testing the current cosmological model (Spergel et al. 2015).

We thank the referee for a careful reading of the manuscript and for insightful suggestions.

Based on observations made with the NASA/ESA Hubble Space Telescope, obtained [from the Data Archive] at the Space Telescope Science Institute, which is operated by the Association of Universities for Research in Astronomy, Inc., under NASA contract NAS 5-26555. These observations are associated with program \#13779.

Support for program \#13779 was provided by NASA through a grant from the Space Telescope Science Institute, which is operated by the Association of Universities for Research in Astronomy, Inc., under NASA contract NAS 5-26555.

A.C. acknowledges grants ASI n.I/023/12/0 "Attivit relative alla fase B2/C per la missione Euclid" and MIUR PRIN 2015 "Cosmology and Fundamental Physics: illuminating the Dark Universe with Euclid." L.C. is supported by DFF 4090-00079.

This work was funded by NASA JWST Interdisciplinary Scientist grants to RAW NAG5-12460 and NNX14AN10G from GSFC.

\section{ORCID iDs}

Sangeeta Malhotra (i) https://orcid.org/0000-0002-9226-5350 Barry Rothberg (ii) https://orcid.org/0000-0003-2283-2185 Norman Grogin (ib https://orcid.org/0000-0001-9440-8872 
Steven L. Finkelstein (1) https://orcid.org/0000-0001-8519-1130

Anton M. Koekemoer (i) https://orcid.org/0000-0002-6610-2048

Rebecca L. Larson (1) https://orcid.org/0000-0003-2366-8858

Lise Christensen (1) https://orcid.org/0000-0001-8415-7547

Andrea Cimatti 10 https://orcid.org/0000-0002-4409-5633

Nimish P. Hathi (1) https://orcid.org/0000-0001-6145-5090

Pascale Hibon (1) https://orcid.org/0000-0003-3726-5494

Darach Watson (1) https://orcid.org/0000-0002-4465-8264

Rogier A. Windhorst (i) https://orcid.org/0000-0001-

8156-6281

Nadia L Zakamska (1) https://orcid.org/0000-0001-6100-6869

Andrew Zirm $\mathbb{1}$ https://orcid.org/0000-0002-6707-275X

\section{References}

Atek, H., Kneib, J.-P., Pacifici, C., et al. 2014, ApJ, 789, 96

Atek, H., Malkan, M., McCarthy, P., et al. 2010, ApJ, 723, 104

Beckwith, S. V. W., Stiavelli, M., Koekemoer, A. M., et al. 2006, AJ, 132, 1729

Bedregal, A. G., Scarlata, C., Henry, A. L., et al. 2013, ApJ, 778, 126

Bertin, E., \& Arnouts, S. 1996, A\&AS, 117, 393

Bouwens, R. J., Illingworth, G. D., Franx, M., \& Ford, H. 2007, ApJ, 670, 928

Bouwens, R. J., Illingworth, G. D., Oesch, P. A., et al. 2015, ApJ, 803, 34

Brammer, G., Ryan, R., \& Pirzkal, N. 2015, Space Telescope WFC3 Instrument Science Report WFC3-2015-17

Brammer, G. B., van Dokkum, P. G., Franx, M., et al. 2012, ApJS, 200, 13 Dahm, S. E. 2005, AJ, 130, 1805

Ellis, R. S., McLure, R. J., Dunlop, J. S., et al. 2013, ApJL, 763, L7

Ferreras, I., Pasquali, A., Khochfar, S., et al. 2012, AJ, 144, 47

Ferreras, I., Pasquali, A., Malhotra, S., et al. 2009, ApJ, 706, 158

Finkelstein, S. L., Papovich, C., Giavalisco, M., et al. 2010, ApJ, 719, 1250

Finkelstein, S. L., Papovich, C., Salmon, B., et al. 2012, ApJ, 756, 164

Finkelstein, S. L., Ryan, R. E., Jr., Papovich, C., et al. 2015, ApJ, 810, 71

Giavalisco, M., Ferguson, H. C., Koekemoer, A. M., et al. 2004, ApJL, 600, L93

Grogin, N. A., Kocevski, D. D., Faber, S. M., et al. 2011, ApJS, 197, 35

Horne, K. 1986, PASP, 98, 609

Illingworth, G. D., Magee, D., Oesch, P. A., et al. 2013, ApJS, 209, 6

Koekemoer, A. M., Ellis, R. S., McLure, R. J., et al. 2013, ApJS, 209, 3

Koekemoer, A. M., Faber, S. M., Ferguson, H. C., et al. 2011, ApJS, 197, 36

Kron, R. G. 1980, ApJS, 43, 305

Kümmel, M., Walsh, J. R., Pirzkal, N., Kuntschner, H., \& Pasquali, A. 2009, PASP, 121, 59

Liu, C. T., \& Green, R. F. 1998, AJ, 116, 1074
Long, K. S., Baggett, S. M., \& MacKenty, J. W. 2013, Space Telescope WFC3 Instrument Science Report 2013-07

MacAlpine, G. M., Smith, S. B., \& Lewis, D. W. 1977, ApJS, 34, 95

Malhotra, S., \& Rhoads, J. E. 2004, ApJL, 617, L5

Markarian, B. E. 1967, Afz, 3, 55

Mayall, N. U. 1936, PASP, 48, 14

McCarthy, P. J., Yan, L., Freudling, W., et al. 1999, ApJ, 520, 548

Momcheva, I. G., Brammer, G. B., van Dokkum, P. G., et al. 2016, ApJS, 225, 27

Oesch, P. A., Bouwens, R. J., Carollo, C. M., et al. 2010, ApJL, 709 L21

Oke, J. B., \& Gunn, J. E. 1983, ApJ, 266, 713

Palmer, H. K. 1903, LicOB, 2, 46

Pasquali, A., Ferreras, I., Panagia, N., et al. 2006, ApJ, 636, 115

Pasquali, A., Pirzkal, N., Walsh, J. R., et al. 2003, in Astronomy, Cosmology and Fundamental Physics, ed. P. A. Shaver, L. DiLella, \& A. Giménez (Berlin: Springer), 471

Pirzkal, N., Pasquali, A., \& Demleitner, M. 2001, STECF, 29, 5

Pirzkal, N., Rothberg, B., Ryan, R., et al. 2013, ApJ, 775, 11

Pirzkal, N., \& Ryan, R. 2017a, Space Telescope WFFC3 Instrument Science Report 2017-01

Pirzkal, N., \& Ryan, R. 2017b, Space Telescope WFFC3 Instrument Science Report 2017-05

Pirzkal, N., Ryan, R., \& Brammer, G. 2016, Space Telescope WFFC3 Instrument Science Report 2017-15

Pirzkal, N., Burgasser, A. J., Malhotra, S., et al. 2009, ApJ, 695, 1591

Pirzkal, N., Rothberg, B., Ly, C., et al. 2013, ApJ, 772, 48

Pirzkal, N., Sahu, K. C., Burgasser, A., et al. 2005, ApJ, 622, 319

Pirzkal, N., Xu, C., Malhotra, S., et al. 2004, ApJS, 154, 501

Robberto, M. 2007, Space Telescope WFC3 Instrument Science Report 2007-10

Ryan, R. E., Hathi, N. P., Cohen, S. H., et al. 2007, ApJ, 668, 839

Sawicki, M. J., Lin, H., \& Yee, H. K. C. 1997, AJ, 113, 1

Schmidt, K., Treu, T., Brammer, G. B., et al. 2014, ApJL, 782, L36

Schmidt, M., Schneider, D. P., \& Gunn, J. E. 1986, ApJ, 306, 411

Smith, M. G. 1975, ApJ, 202, 591

Smith, M. G. 1978, VA, 22, 321

Spergel, D. N., Gehrels, N., Baltay, C., et al. 2015, arXiv:1503.03757

Stern, D., Yost, S. A., Eckart, M. E., et al. 2005, ApJ, 619, 12

Straughn, A. N., Meurer, G. R., Pirzkal, N., et al. 2008, AJ, 135, 1624

Tilvi, V., Pirzkal, N., Malhotra, S., et al. 2016, ApJL, 827, L14

Treu, T., Schmidt, K. B., Brammer, G. B., et al. 2015, ApJ, 812, 114

Valdes, F. G., Campusano, L. E., Velasquez, J. D., \& Stetson, P. B. 1995, PASP, 107, 1119

Wasilewski, A. J. 1983, ApJ, 272, 68

Whitaker, K. E., van Dokkum, P. G., Brammer, G., et al. 2013, ApJL, 770, L39

Windhorst, R. A., Cohen, S. H., Hathi, N. P., et al. 2011, ApJS, 193, 27 